\title{
Suppression of the Kapitza instability in confined falling liquid films
}

\begin{tabular}{|r|l|}
\hline Journal: & Journal of Fluid Mechanics \\
\hline Manuscript ID & JFM-18-S-0685.R1 \\
\hline mss type: & JFM Papers \\
\hline Complete List of Authors: & $\begin{array}{l}\text { Lavalle, Gianluca; Laboratoire d'Informatique pour la Mecanique et les } \\
\text { Sciences de I'Ingenieur, } \\
\text { Li, Yiqin; Fluides Automatique et Systemes Thermiques } \\
\text { Mergui, Sophie; Fluides Automatique et Systemes Thermiques } \\
\text { Grenier, Nicolas; Laboratoire d'Informatique pour la Mecanique et les } \\
\text { Sciences de I'Ingenieur } \\
\text { Dietze, Georg; CNRS, Laboratoire FAST - UMR 7608 }\end{array}$ \\
\hline Keyword: & $\begin{array}{l}\text { Thin films < Interfacial Flows (free surface), Instability, Gas/liquid flows < } \\
\text { Multiphase and Particle-laden Flows }\end{array}$ \\
\hline & \\
\hline
\end{tabular}

\section{SCHOLARONE}




\title{
Suppression of the Kapitza instability in confined falling liquid films
}

\author{
Gianluca Lavalle ${ }^{1} \dagger$, Yiqin $\mathbf{L i}^{2}$, Sophie Mergui ${ }^{2}$, Nicolas Grenier ${ }^{1}$, and \\ Georg F. Dietze 2 \\ ${ }^{1}$ Laboratoire LIMSI, CNRS, Univ. Paris-Sud, Université Paris-Saclay, F-91405, Orsay, France \\ ${ }^{2}$ Laboratoire FAST, Univ. Paris-Sud, CNRS, Université Paris-Saclay, F-91405, Orsay, France
}

(Received $\mathrm{xx}$; revised $\mathrm{xx}$; accepted $\mathrm{xx}$ )

We revisit the linear stability of a falling liquid film flowing through an inclined narrow channel in interaction with a gas phase. We focus on a particular region of parameter space, small inclination and very strong confinement, where we have found the gas to strongly stabilize the film, up to the point of fully suppressing the long-wave interfacial instability attributed to Kapitza (Kapitza, Zh. Eksp. Teor. Fiz. 18, 1948). The stabilization occurs both when the gas is merely subject to an aerostatic pressure difference, i.e. when the pressure difference balances the weight of the gas column, and when it flows counter-currently. In the latter case, the degree of stabilization increases with the gas velocity. Our investigation is based on a numerical solution of the Orr-Sommerfeld temporal linear stability problem as well as stability experiments that clearly confirm the observed effect. We quantify the degree of stabilization by comparing the linear stability threshold with its passive-gas limit, and perform a parametric study, varying the relative confinement, the Reynolds number, the inclination angle and the Kapitza number. For example, we find a $30 \%$ reduction of the cut-off wavenumber of the instability for a water film in contact with air, flowing through a channel inclined at 3 degrees and of height 2.8 times the film thickness. We also identify the critical conditions for the full suppression of the instability in terms of the governing parameters. The stabilization is caused by the strong confinement of the gas, which produces perturbations of the adverse interfacial tangential shear stress that are shifted by half a wavelength with respect to the wavy film surface. This tends to reduce flow rate variations within the film, thus attenuating the inertia-based driving mechanism of the Kapitza instability.

\section{Key words:}

\section{Introduction}

We consider a liquid film falling along the bottom wall of an inclined narrow channel, under the action of gravity and in interaction with a laminar gas flow (see figure 1). We distinguish two scenarios for the gas flow: (i) either the gas is subject to an aerostatic pressure gradient, i.e. a pressure difference which balances the weight of the gas column; (ii) or the gas flows counter-currently to the liquid film at an imposed flow rate. Examples of the velocity profiles corresponding to these two scenarios are depicted in figure 2 . We revisit the linear stability of this flow by numerically solving the Orr-Sommerfeld temporal linear stability problem and by performing stability experiments. We are

$\dagger$ Email address for correspondence: gianluca.lavalle@limsi.fr 


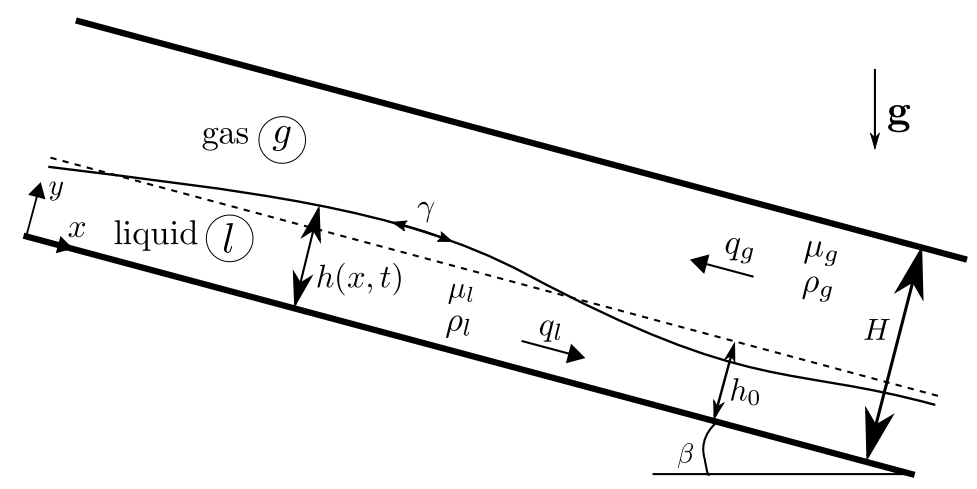

FiguRE 1. Sketch of the considered problem: a liquid film falling down an inclined wall whilst interacting with a strongly-confined gas phase. The gas is either subject to an aerostatic pressure difference or flows counter-currently at an imposed flow rate. The flow rates $q_{l}$ and $q_{g}$ and the heights $h, h_{0}$ and $H$ are dimensionless.

particularly interested in how the stability of the falling film is affected by the gas phase in the case of strong confinement. Among the susceptible instability modes of the film (Boomkamp \& Miesen 1996), we focus solely on the convective long-wave interfacial mode (Trifonov 2017) known as Kapitza instability (Kapitza 1948). For example, short-wave modes occurring in the liquid (Floryan et al. 1987) and/or gas (Schmidt et al. 2016) do not play a role in our study.

We start by recalling some theoretical results for the case of a liquid film falling in a passive atmosphere. Benjamin (1957) and Yih (1963) solved the associated OrrSommerfeld temporal linear stability problem and proved that a vertical liquid film is always unstable to long-wave surface disturbances. Further, they determined the threshold of this long-wave instability in terms of the critical Reynolds number $R e_{c r}=$ $5 / 6 \cot \beta$, which depends on the inclination angle $\beta$. The instability mechanism is due to inertia, while the role of the longitudinal component of gravity is to advect and steepen the wave, whereas surface tension stabilizes the film. The way inertia destabilizes the film has been explained either via the shift between the vorticity and the displacement of the interface (Kelly et al. 1989; Smith 1990; Kalliadasis et al. 2012), following the argument introduced by Hinch (1984) for two-layer Couette flows, or via the inertia-related lag of flow-rate perturbations with respect to film-thickness perturbations (Dietze 2016).

Investigations of the effect of an active outer phase on the stability of the film started with works dedicated to a slightly different configuration. Yih (1967) investigated the pressure-driven channel flow of two co-current fluid layers and showed that a viscosity contrast between layers of equal density and thickness causes a long-wave interfacial instability. This work was extended by Yiantsios \& Higgins (1988), who accounted for a density contrast and a non-unity thickness ratio, and included gravity as well as surface tension. More recently, spatio-temporal linear stability analysis was applied to this problem (Valluri et al. 2010; Ó Náraigh et al. 2013).

By additionally accounting for an inclination of the channel, Tilley et al. (1994) were able to investigate (among other scenarios) the stability of a falling water film in contact with air, which is the configuration we are interested in. In fact, their temporal OrrSommerfeld linear stability problem is exactly the same as the one we solve here (see §2). The authors argued that confining the gas phase by an upper wall is likely to stabilize the falling film through an adverse shear stress at the liquid/gas interface. However, their calculations for the water-air system led them to conclude that this effect is negligible. 
Indeed, the minimum cut-off wavenumber upon varying the total flow rate changed only slightly with the channel height. In our current study, we have come to a different conclusion. We find that the cut-off wavenumber can be reduced up to the point of fully suppressing the Kapitza instability by diminishing the channel height at a fixed liquid flow rate. This holds both when the gas is subject to an aerostatic pressure difference and when it flows counter-currently.

A number of studies have investigated the effect of a turbulent counter-current gas flow on the stability of a falling liquid film. By applying a spatio-temporal analysis, Vellingiri et al. (2015) found a convective/absolute/upward-convective transition of the instability when increasing the interfacial shear stress exerted by the gas (conditions studied in our current manuscript are far removed from this transition). The authors also found that the cut-off wavenumber of the convective instability may either diminish, increase, or display a non-monotonic behaviour with increasing gas shear stress. Trifonov (2017) made similar observations (also for turbulent gas flow) based on extensive temporal stability calculations, where the inclination angle, confinement, and liquid Reynolds number where additionally varied. For the strong confinement levels on which we mainly focus in our current study, we have observed only a monotonic decrease of the cut-off wavenumber with increasing gas shear stress. However, we have checked that we recover the same qualitative behaviour as Trifonov (2017) and Vellingiri et al. (2015) when the confinement level is decreased, notwithstanding that the gas flow in our case is laminar and not turbulent.

We proceed by discussing several related experimental works that have focused on the linear stability of falling liquid films. Krantz \& Goren (1971) studied liquid films flowing down a strongly inclined plane at low Reynolds numbers. They imposed inlet disturbances of controlled amplitude and frequency, and measured wave celerities as well as spatial growth rates. Later, Pierson \& Whitaker (1977) and Alekseenko et al. (1985) studied liquid films flowing down the outside of a vertical cylinder. They measured the wavelength, wave celerity and spatial growth rate for the fastest growing waves over a large range of Reynolds numbers, showing reasonable agreement with the theory developed in previous studies (Pierson \& Whitaker 1977; Whitaker 1964).

Liu et al. (1993) studied liquid films falling down a weakly inclined plane at moderate Reynolds numbers. By imposing a small-amplitude perturbation of controlled frequency on the liquid flow rate, the authors were able to measure the critical Reynolds number in terms of the inclination angle, the cut-off wavenumber as a function of the Reynolds number, and the dispersion curves of the spatial growth rate and wave celerity. Their experimental data agreed with the neutral stability curve of Anshus \& Goren (1966).

Alekseenko et al. (2009) investigated the additional effect of an active gas phase by measuring the spatial growth rate of small-amplitude surface waves excited on a liquid film falling down the inner surface of a vertical tube in interaction with a co- or countercurrent turbulent gas flow. In particular, the authors found that increasing the countercurrent gas flow reduces the cut-off wavenumber of the Kapitza instability, while the maximal growth rate is increased. Thus, shorter waves are stabilized while longer waves are amplified. Nonlinearly, this manifests itself in the attenuation of the precursory capillary ripples typically forming on wavy falling liquid films (Trifonov 2010). This has been also detected experimentally by Kofman et al. (2017). We observe a different behaviour in our current study. For the strong confinement levels considered, we find that the linear growth rate is decreased at all unstable wavenumbers when the counter-current gas velocity is increased. However, in the case of a weaker confinement, we recover the same qualitative behaviour as Alekseenko et al. (2009) for the laminar gas flow conditions studied here (see section 4.3). 
Our manuscript is structured as follows. In $\S 2$, we write the Orr-Sommerfeld temporal linear stability eigenvalue problem governing the stability of the falling film; the set-up and measurement methodology for the linear stability experiments are presented in $\S 3$; in $\S 4$ we present the results of our stability analysis focusing on the stabilizing effect of confinement in terms of the liquid Reynolds number, the inclination angle, the Kapitza number, and the velocity of a counter-current gas flow; the mechanism responsible for the stabilization induced by the confinement is presented in $\S 5$; finally, concluding remarks are presented in $\S 6$.

\section{Governing equations and linear stability problem}

For the configuration depicted in figure 1, the governing equations in dimensionless form read as (subscripts $l$ and $g$ are phase indicators):

$$
\begin{gathered}
\nabla \cdot \mathbf{u}_{l}=0, \quad \nabla \cdot \mathbf{u}_{g}=0, \\
D_{t} \mathbf{u}_{l}=-\Pi_{\rho} \nabla p_{l}+\mathbf{B}+\Delta \mathbf{u}_{l}, \\
D_{t} \mathbf{u}_{g}=-\nabla p_{g}+\mathbf{B}+\Pi_{\mu} \Pi_{\rho}^{-1} \Delta \mathbf{u}_{g},
\end{gathered}
$$

where $\Pi_{\rho}=\rho_{g} / \rho_{l}$ and $\Pi_{\mu}=\mu_{g} / \mu_{l}$ designate the density and viscosity ratios. Here $\mathbf{u}$ is the velocity vector of components $(u, v)$ along $(x, y), p$ the pressure, $\Delta$ the Laplace operator, $D_{t}$ the material derivative, and $\mathbf{B}=(\sin \beta,-\cos \beta)$ the inclination vector with the inclination angle $\beta$. For non-dimensionalization, we have employed reference scales obtained from balancing viscous drag and gravity, i.e. $\mathcal{L}=\nu_{l}^{2 / 3} g^{-1 / 3}, \mathcal{U}=\left(\nu_{l} g\right)^{1 / 3}$ and $\mathcal{T}=\mathcal{L} / \mathcal{U}$ for length, velocity, and time, and $\rho_{g} \mathcal{U}^{2}$ for pressure. The reader is warned that the full gravitational acceleration $g$ rather than its streamwise projection $g_{x}=g \sin \beta$ is used here, in contrast to Ruyer-Quil \& Manneville (2000). This way, the scales are independent of the control parameters. As a result of our scaling, the Reynolds and Froude numbers do not appear explicitly in (2.1). We thus introduce the following definition for the Reynolds number in the liquid and gas:

$$
R e_{l}=\frac{\tilde{q}_{l}}{\nu_{l}}, \quad R e_{g}=\frac{\tilde{q}_{g}}{\nu_{g}},
$$

where $\tilde{q}_{l}$ and $\tilde{q}_{g}$ are the dimensional liquid and gas flow rates per unit width (the tilde will denote dimensional quantities throughout the manuscript).

The boundary conditions at the walls read:

$$
\mathbf{u}(0)=0, \quad \mathbf{u}(H)=0 .
$$

The kinematic and dynamic coupling conditions at the interface $y=h$ are:

$$
\begin{gathered}
u_{l}=u_{g} \\
\left(\boldsymbol{S}_{l} \cdot \mathbf{n}\right) \cdot \mathbf{t}=\Pi_{\mu}\left(\boldsymbol{S}_{g} \cdot \mathbf{n}\right) \cdot \mathbf{t} \\
\left(\boldsymbol{S}_{l} \cdot \mathbf{n}\right) \cdot \mathbf{n}+\kappa W e=\Pi_{\rho}\left(\boldsymbol{S}_{g} \cdot \mathbf{n}\right) \cdot \mathbf{n}
\end{gathered}
$$

where $W e=\gamma\left(\rho_{l} \mathcal{L U}^{2}\right)^{-1}$ is the Weber number with surface tension $\gamma$. Note that, due to the chosen scaling, our Weber number is identical to the Kapitza number $K a=$ $\gamma\left(\rho_{l} g^{1 / 3} \nu_{l}^{4 / 3}\right)^{-1}$ which prevails in the literature on falling films. In $(2.4 \mathrm{~b})$ and $(2.4 \mathrm{c})$, $\boldsymbol{S}=\boldsymbol{T}-p \boldsymbol{I}$ is the stress tensor, $\boldsymbol{T}$ the viscous stress tensor and $\boldsymbol{I}$ the identity matrix. The normal and tangential vectors to the interface are defined as:

$$
\mathbf{n}=\frac{\left[-\partial_{x} h, 1\right]}{\sqrt{1+\left(\partial_{x} h\right)^{2}}}, \quad \mathbf{t}=\frac{\left[1, \partial_{x} h\right]}{\sqrt{1+\left(\partial_{x} h\right)^{2}}},
$$



whereas $\kappa=\nabla \cdot \mathbf{n}=-\partial_{x x} h\left[1+\left(\partial_{x} h\right)^{2}\right]^{-3 / 2}$ is the curvature of the interface. The kinematic condition at the interface reads:

$$
\partial_{t} h+u_{l} \partial_{x} h=v_{l}
$$

In our analysis, we will vary the relative confinement of the film, which is defined as:

$$
\eta=\frac{H}{h_{0}},
$$

where $h_{0}$ designates the film thickness of the primary flow and $H$ the gap height. It constitutes one of the control parameters of the problem, together with the inclination angle $\beta$ and the flow rates $q_{l}$ and $q_{g}$.

We perform a temporal stability analysis following the works of Yih (1967), Yiantsios \& Higgins (1988) and Tilley et al. (1994). We start by writing the base flow velocity profiles:

$$
\begin{aligned}
U_{l} & =K_{l}\left(\frac{y^{2}}{2}-h_{0} y\right)+\Pi_{\rho} T_{t} y \\
U_{g} & =K_{g} \frac{h_{0}^{2}}{2}\left(\eta-\frac{y}{h_{0}}\right)\left(2-\eta-\frac{y}{h_{0}}\right)-\frac{\Pi_{\rho}}{\Pi_{\mu}} T_{t} h_{0}\left(\eta-\frac{y}{h_{0}}\right) .
\end{aligned}
$$

The constants $K_{l}$ and $K_{g}$ are defined as:

$$
\begin{gathered}
K_{l}=\Pi_{\rho} \partial_{x} P-\sin \beta\left(1-\Pi_{\rho} M\right), \\
K_{g}=\Pi_{\rho} \Pi_{\mu}^{-1}\left[\partial_{x} P-\sin \beta(1-M)\right],
\end{gathered}
$$

where $\partial_{x} P$ is the driving pressure gradient and $M=\Delta p / \Delta p^{a}$ relates the driving pressure difference $\Delta p$ to the aerostatic pressure difference $\Delta p^{a}=\Lambda \sin \beta, \Lambda$ designating the (dimensionless) wavelength. The coefficient $M$ allows to distinguish between the two studied scenarios for the gas phase (see figure 2): (i) an aerostatic pressure difference, where $M=1$; (ii) a counter-current gas flow, where $M>1$.

The interfacial tangential shear stress appearing in (2.8) is scaled with $\rho_{g} \mathcal{U}^{2}$ and reads:

$$
T_{t}=\frac{h_{0}}{2 \Pi_{\rho}}\left[K_{l}-K_{g}(\eta-1)^{2}\right]\left[1+\frac{1}{\Pi_{\mu}}(\eta-1)\right]^{-1},
$$

while the pressure profiles read:

$$
\begin{gathered}
P_{l}=\left.P\right|_{h_{0}}(x=0)+x \partial_{x} P+\Pi_{\rho}^{-1} \cos \beta\left(h_{0}-y\right), \\
P_{g}=\left.P\right|_{h_{0}}(x=0)+x \partial_{x} P+\cos \beta\left(h_{0}-y\right),
\end{gathered}
$$

where $\left.P\right|_{h_{0}}(x=0)$ is an arbitrary reference pressure at $y=h_{0}$ and $x=0$.

We linearly perturb the primary flow as follows:

$$
u=U+u^{\star}, \quad v=v^{\star}, \quad p=P+p^{\star},
$$

where upper case letters refer to the base flow, while the star symbol denotes a smallamplitude perturbation. We introduce the stream-function perturbations $\phi^{\star}$ in the liquid phase and $\psi^{\star}$ in the gas phase, satisfying:

$$
\begin{array}{ll}
u_{l}^{\star}=\partial_{y} \phi^{\star}, & v_{l}^{\star}=-\partial_{x} \phi^{\star}, \\
u_{g}^{\star}=\partial_{y} \psi^{\star}, & v_{g}^{\star}=-\partial_{x} \psi^{\star},
\end{array}
$$

and seek solutions of the form:

$$
\begin{aligned}
& \left\{\phi^{\star}, p_{l}^{\star}\right\}=\left\{\hat{\phi}(y), \hat{p}_{l}(y)\right\} \exp [i \alpha(x-c t)], \\
& \left\{\psi^{\star}, p_{g}^{\star}\right\}=\left\{\hat{\psi}(y), \hat{p}_{g}(y)\right\} \exp [i \alpha(x-c t)],
\end{aligned}
$$




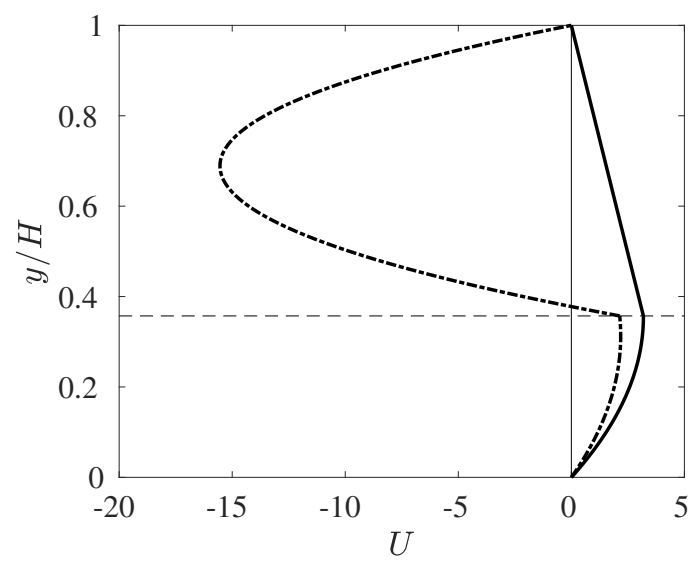

Figure 2. Base-state velocity profiles for an air-water flow: $\beta=3^{\circ}, \eta=2.8(\tilde{H}=1.5 \mathrm{~mm})$. The flat dashed line represents the interface, the solid line the aerostatic case $(M=1$ in $(2.9))$ with $R e_{l}=23.6$, whereas the dashed-dotted line the counter-current case $(M=100$ in (2.9)) with $R e_{l}=17.1$ and $R e_{g}=-12.8$.

where $\alpha \in \mathbb{R}$ is the wavenumber and $c=c_{r}+i c_{i}$ the complex wave celerity, whereas the hat symbol denotes the amplitudes of the corresponding perturbations. Substituting (2.14) into (2.1) linearized around the base flow, and eliminating the pressure in $(2.1 b)$ by substituting (2.1c), we obtain the well-known Orr-Sommerfeld equations:

$$
\begin{aligned}
\hat{\phi}^{\prime \prime \prime \prime}-2 \alpha^{2} \hat{\phi}^{\prime \prime}+\alpha^{4} \hat{\phi} & =i \alpha\left[\left(U_{l}-c\right)\left(\hat{\phi}^{\prime \prime}-\alpha^{2} \hat{\phi}\right)-U_{l}^{\prime \prime} \hat{\phi}\right], \\
\hat{\psi}^{\prime \prime \prime \prime}-2 \alpha^{2} \hat{\psi}^{\prime \prime}+\alpha^{4} \hat{\psi} & =i \alpha\left[\left(U_{g}-c\right)\left(\hat{\psi}^{\prime \prime}-\alpha^{2} \hat{\psi}\right)-U_{g}^{\prime \prime} \hat{\psi}\right],
\end{aligned}
$$

subject to the boundary conditions:

$$
\begin{gathered}
\hat{\phi}(0)=0, \quad \hat{\phi}^{\prime}(0)=0, \\
\hat{\psi}(H)=0, \quad \hat{\psi}^{\prime}(H)=0,
\end{gathered}
$$

where the prime symbol denotes differentiation with respect to $y$. Linearization of the coupling conditions (2.4) must account for the perturbation of the variable fields as well as a perturbation of the position at which the condition is applied, i.e. at the interface. Consider the perturbed interfacial value $\left.\{f\}\right|_{h}$ of the variable $f(x, y, t)$ with base state $F(y)$. This can be decomposed into:

$$
\left.\{f\}\right|_{h}=\left.\{F\}\right|_{h_{0}}+\left.\left\{f^{\star}\right\}\right|_{h_{0}}+\left.\left\{F^{\prime}\right\}\right|_{h_{0}} h^{\star},
$$

where $f^{\star}(x, y, t)=\hat{f}(y) \exp [i \alpha(x-c t)]$ stems from the perturbation of the variable field and $\left\{F^{\prime}\right\} \mid h_{0} h^{\star}$ is the contribution from changing the position of the film surface within this field.

Applying (2.18) to the coupling conditions (2.4) leads to:

$$
\begin{gathered}
\hat{\phi}^{\prime}+U_{l}^{\prime} \hat{\phi} \underline{c}^{-1}=\hat{\psi}^{\prime}+U_{g}^{\prime} \hat{\phi} \underline{c}^{-1} \\
\hat{\phi}^{\prime \prime}+U_{l}^{\prime \prime} \hat{\phi}^{-1}+\alpha^{2} \hat{\phi}=\Pi_{\mu}\left[\hat{\psi}^{\prime \prime}+U_{g}^{\prime \prime} \hat{\phi} \underline{c}^{-1}+\alpha^{2} \hat{\psi}\right] \\
2 \alpha^{2} \hat{\phi}^{\prime}-i \alpha \Pi_{\rho}\left[\hat{p}_{l}+P_{l}^{\prime} \hat{\phi} \underline{c}^{-1}\right]+W e i \alpha^{3} \hat{\phi} \underline{c}^{-1}=2 \alpha^{2} \Pi_{\mu} \hat{\psi}^{\prime}-i \alpha \Pi_{\rho}\left[\hat{p}_{g}+P_{g}^{\prime} \hat{\phi} \underline{c}^{-1}\right](2.1
\end{gathered}
$$

where all quantities are evaluated at $y=h_{0}$ and where we have introduced $\underline{c}=c-\left.U\right|_{h_{0}}$ 
and used the relation:

$$
h-h_{0}=h^{\star}=\hat{\phi} \underline{c}^{-1} \exp [i \alpha(x-c t)],
$$

which follows directly from (2.6) together with (2.18). The pressure perturbation amplitudes $\hat{p}_{l}$ and $\hat{p}_{g}$ in $(2.19 c)$, evaluated at $y=h_{0}$, can be recovered directly from (2.1):

$$
\begin{aligned}
& \hat{p}_{l}=\Pi_{\rho}^{-1}\left[\underline{c} \hat{\phi}^{\prime}+U_{l}^{\prime} \hat{\phi}\right]+\left(i \alpha \Pi_{\rho}\right)^{-1}\left(\hat{\phi}^{\prime \prime \prime}-\alpha^{2} \hat{\phi}^{\prime}\right), \\
& \hat{p}_{g}=\left[\underline{c} \hat{\psi}^{\prime}+U_{g}^{\prime} \hat{\psi}\right]+\Pi_{\mu}\left(i \alpha \Pi_{\rho}\right)^{-1}\left(\hat{\psi}^{\prime \prime \prime}-\alpha^{2} \hat{\psi}^{\prime}\right) .
\end{aligned}
$$

The Orr-Sommerfeld problem (2.15)-(2.21) is identical to the problem studied by Tilley et al. (1994). We solve it by means of continuation using a code based on the continuation software AUTO-07p (Doedel 2008). This code was previously used in Dietze \& RuyerQuil (2013). The continuation is started from the asymptotic limit $\alpha=0$, for which $c_{r}$ and $c_{i}=0$ are known analytically, and continued by varying one of the control parameters while calculating $c_{r}$ and $c_{i}$. We have validated our numerical procedure by comparing stability results with those of Brevdo et al. (1999) for falling films in a passive atmosphere, those of Yiantsios \& Higgins (1989) in the asymptotic limit of long waves, and those of Yiantsios \& Higgins (1988) and Tilley et al. (1994) for a pressure-driven horizontal liquid-gas flow. These validations are presented in the appendix A.

We point out that the considered gas flow rates are far from the convective-absolute transition studied by Vellingiri et al. (2015), Schmidt et al. (2016), and Lavalle et al. (2017), thus no spatio-temporal analysis is required here. For additional security, we have validated our stability calculations with our own experiments, which are described in the next section 3. Due to the convective nature of the instability, we measure a spatial growth rate in our experiments, whereas our stability calculations yield temporal growth rates (in a reference frame moving at the wave celerity). Both approaches yield the same stability threshold and most of our comparisons between experiments and linear stability analysis concern this stability threshold. However, in one instance (figure $9 b$ ), we compare experimental and numerical growth rate dispersion curves and for this we apply the so-called Gaster transformation (Gaster 1962) that relates temporal and spatial growth rates:

$$
\alpha_{r} c_{i}=-\zeta\left(c_{r}+\alpha_{r} \partial_{\alpha_{r}} c_{r}\right),
$$

where $\zeta$ is the spatial growth rate and $\alpha_{r} c_{i}$ the temporal one. This transformation is valid when the spatial and temporal growth rates are small. This is generally the case for falling liquid films, as shown by Brevdo et al. (1999), who compared spatial growth rates obtained directly from a spatio-temporal analysis with values obtained by applying the Gaster transformation to temporal stability results, and showed that the error is smaller than $0.1 \%$. In the single instance where we have applied the Gaster transformation (figure 9b), flow conditions are comparable to those in Brevdo et al. (1999).

\section{Experiments}

Our experimental apparatus, sketched in figure 3, has been employed in previous studies (Kofman 2014; Kofman et al. 2017) and has been modified here to impose a much stronger confinement of the gas phase above the falling liquid film (figure 4). The liquid film flows down an inclined glass plate $(150 \mathrm{~cm}$ long, $27 \mathrm{~cm}$ wide, $5 \mathrm{~mm}$ thick) fixed in a framework mounted on rubber feet to reduce the influence of environmental vibrations. The inclination angle $\beta$ can be changed in the range $0^{\circ}-20^{\circ}$ and is measured using an inclinometer with a precision of $0.05^{\circ}$.

A gear pump conveys the liquid from a collection tank located at the end of the plate to 


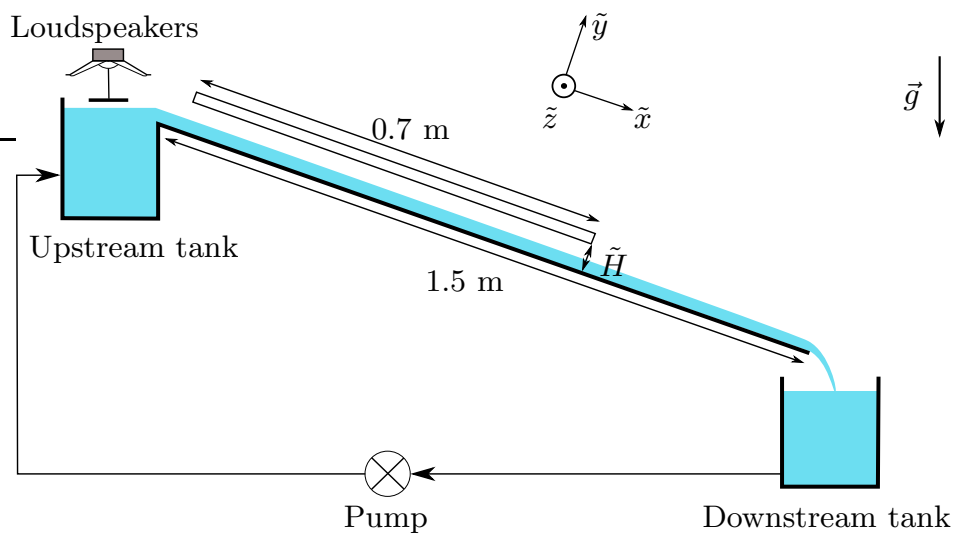

Figure 3. Sketch of the experimental set-up.

(a)

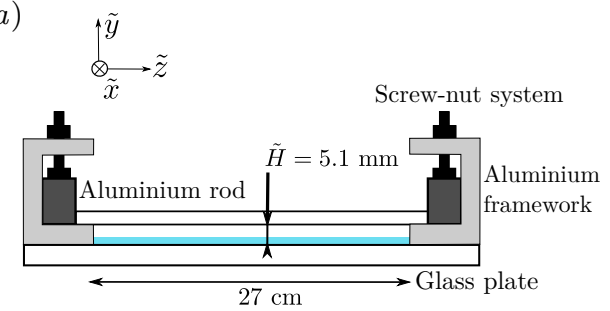

(b)

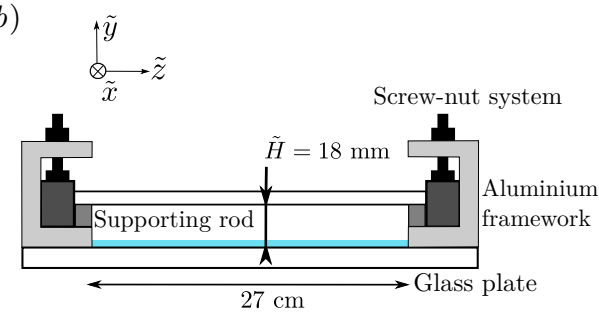

Figure 4. Sketch of the channel cross-section: (a) confined and (b) "unconfined" configuration.

an inlet tank, from which the liquid overflows onto the glass plate. The inlet tank is filled with several layers of glass beads in order to homogenize the entering flow. A temporal periodic forcing of the film is introduced at the inlet to trigger sinusoidal surface waves of prescribed frequency and amplitude. This is achieved by a vibrating aluminum plate, which is fixed to the membrane of two loudspeakers and generates harmonic vibrations above the liquid surface over the whole width of the film (Kofman et al. 2017).

At a position $5 \mathrm{~cm}$ from the inlet, the falling film enters a region where the gas phase is confined by an upper $5 \mathrm{~mm}$ thick glass plate of length $70 \mathrm{~cm}$ and width $27 \mathrm{~cm}$ (see figure 3 ). In our study, the gap height $\tilde{H}$ of the channel formed between the two glass plates was set to two different values: (i) $\tilde{H}=5.1 \mathrm{~mm} \pm 0.1 \mathrm{~mm}$, which we call the confined case; (ii) $\tilde{H}=18 \mathrm{~mm}$, which we loosely refer to as the "unconfined" case. The shift from one case to the other is achieved by raising the top glass plate upon supporting rods (see figure 4). For the confined configuration, the uniformity of the gap height was verified both in the streamwise and the transverse directions.

Water is used as working liquid. The temperature of the liquid is measured in the inlet and downstream tanks, and the surface tension is regularly monitored by measuring a water sample in a pending drop tensiometer. The liquid flow rate is fixed large enough that the film is unstable but small enough that the film surface without inlet excitation is virtually flat in the measurement section (i.e. surface waves are undetectable). The experimental control parameters and the range of variation of the physical parameters are specified in tables 1 and 2 .

To experimentally characterize the interfacial instability of the falling film, a onepoint measurement of the film thickness time trace is performed using the CCI (Confocal Chromatic Imaging) technique (Cohen-Sabban et al. 2001). The sensor model used in our experiments (STIL CCS-PRIMA CL4) allows us to detect two interface locations 


$\begin{array}{lcc}\text { Control parameter } & \text { Notation } & \text { Domain of variation } \\ \text { Inclination angle } & \beta & 1.69^{\circ} \text { and } 3.05^{\circ} \\ \text { Reynolds number } & R e_{l} & 17.7-41.6 \\ \text { Forcing frequency } & \tilde{f} & 1.8-8.4 \mathrm{~Hz}\end{array}$

TABLE 1. Range of variation of the control parameters for the experiments.

$\begin{array}{lcc}\text { Physical property } & \text { Notation } & \text { Range of variation } \\ \text { Density } & \rho_{l} & 997-999 \mathrm{~kg} / \mathrm{m}^{3} \\ \text { Kinematic viscosity } & \nu_{l} & 8.9-11.1 \times 10^{-7} \mathrm{~m}^{2} / \mathrm{s} \\ \text { Surface tension } & \gamma & 71.1-71.7 \mathrm{mN} / \mathrm{m}\end{array}$

TABLE 2. Physical properties of water at ambient temperature and their range of variation during our experiments.

simultaneously (the glass-water and the water-air interface) with a precision of $250 \mathrm{~nm}$ and an acquisition frequency up to $2 \mathrm{kHz}$. The CCI probe is mounted on a linear translation stage in order to enable measurements along the streamwise axis of the channel. The film thickness is measured at mid-width of the channel and at different longitudinal locations from the inlet. Measurements in the transverse direction were also carried out to check the uniformity of the film thickness, at sufficient distance from the lateral boundaries.

Figure 5 displays the streamwise evolution of the time-averaged film thickness $\tilde{h}_{m}$ along the central axis of the channel, in the unconfined configuration. Two wave regimes are represented: (i) a virtually flat film, observed without inlet excitation (empty circles), and (ii) periodically-excited waves, observed with inlet forcing (filled triangles).

The actual flow rate of the falling liquid film is deduced from a film thickness measurement without inlet excitation, i.e. when the film surface is virtually flat. Indeed, for a fully-developed waveless film, the film thickness $h_{0}$ and the Reynolds number $R e_{l}$ are linked through the base flow velocity profile (2.8) subject to an aerostatic pressure difference:

$$
R e_{l}=-K_{l} \frac{h_{0}^{3}}{3}+T_{t} \frac{h_{0}^{2}}{2},
$$

where $K_{l}$ is defined in (2.9a) (using $M=1$ for the aerostatic scenario) and the interfacial tangential shear stress $T_{t}$ in (2.10). By measuring the film thickness without inlet excitation in the developed region of the film (where $\tilde{h}_{m}$ in figure 5 no longer changes), we obtain $R e_{l}$ from (3.1) with $h_{0}=h_{m}$.

The same waveless signal also serves a second purpose. It is compared to the film thickness measured with inlet excitation to determine an upper limit for the excitation amplitude below which the excited surface waves can be considered linear, i.e. governed by the linearized Navier-Stokes equations (2.15). In that case, the film thickness perturbation is sinusoidal in time (2.20) and thus the time-average of the perturbed film thickness $h_{0}+h^{\star}$ corresponds to the thickness of the waveless film $h_{0}$. The excitation amplitude in the experiments was limited to values where this condition was satisfied in order to allow comparisons with the linear stability analysis.

Consequently, the waves excited in our experiments were sinusoidal (see also inset in figure $6 a$ ), and their amplitude $\tilde{A}$, defined here as the peak-to-peak value of the time 


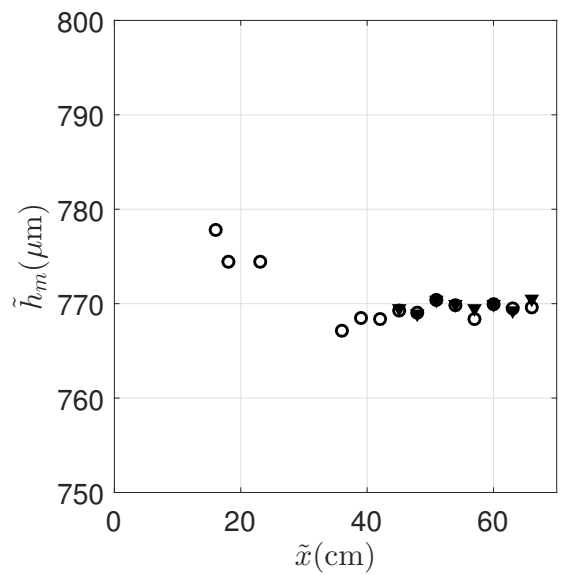

Figure 5. Streamwise evolution of the time-averaged film thickness $\tilde{h}_{m}$ along the central axis of the channel, with inlet excitation $(\tilde{f}=4.2 \mathrm{~Hz}$, filled triangles $)$ and without inlet excitation (empty circles). Parameters: $\beta=1.69^{\circ}, R e_{l}=41.5$, unconfined configuration $\tilde{H}=18 \mathrm{~mm}$.

signal, can be obtained in a statistically robust manner by performing a discrete Fourier transform of the film thickness time trace measured with the CCI system.

The frequency resolution $\Delta f=f_{p} / N_{p}$ of the discrete Fourier analysis is limited by the acquisition frequency $f_{p}$ and the number of sampling points $N_{p}$. In all our measurements, a minimum of 5 series of at least 80 periods of the forcing mode was acquired. Thereby, $f_{p}$ and $N_{p}$ were chosen such that $\Delta f \leqslant 0.025 \mathrm{~Hz}$. An example of the CCI signal and the corresponding modulus of the discrete Fourier transform are shown in panels $6 a$ and $6 b$. Here the forcing frequency is $3 \mathrm{~Hz}\left(\beta=1.69^{\circ}, R e_{l}=34.8\right)$. The predominant peak appearing in figure $6 b$ indicates that the signal is mono-periodic with a frequency equal to the forcing frequency, as expected. The amplitude of this mode is directly obtained from the peak height, provided that the signal contains an integer number of periods of the relevant mode (Bergé et al. 1988). Under this condition, the amplitude is four times the height of the peak (here $\tilde{A}=4 \times 4.4=17.6 \mu \mathrm{m}$ ).

In panels $6 c$ and $6 d$ we have additionally represented a CCI signal and the corresponding spectrum obtained without inlet forcing for the same angle and Reynolds number as in panels $6 a$ and $6 b$. The spectrum exhibits peaks of very small amplitude (the scale has been divided by 10 compared to panel $6 b$ ) that are the signature of the intrinsic noise of the set-up. The bump around $3 \mathrm{~Hz}$ and the low-frequency peaks are intrinsic to the set-up as they are present in all our signals. The peak observed at $4.2 \mathrm{~Hz}$ is most-probably due to the pump, the frequency of which increases with the Reynolds number, i.e. with the flow rate delivered by the pump. The noise level is low enough that the quality of the signal measured with inlet forcing is not deteriorated.

To investigate the linear stability of the film, we measure the spatial evolution of the wave amplitude along the channel. This allows us to obtain: (i) the spatial growth rate of the instability as a function of the forcing frequency for a given inclination angle and Reynolds number, i.e. the dispersion relation; (ii) the cut-off frequency of the instability, $\tilde{f}_{c}$, as a function of the Reynolds number for a given angle $\beta$, i.e. the neutral stability curve $\tilde{f}_{c}\left(R e_{l}\right)$.

The spatial growth rate for a given frequency at a fixed (supercritical) Reynolds number is determined as follows. In the linear regime, the wave amplitude grows exponentially as a function of $\tilde{x}, \tilde{A}(\tilde{x}) \propto \exp (\tilde{\zeta} \tilde{x})$ where $\tilde{\zeta}$ is the spatial growth rate and $\tilde{x}$ is the streamwise location of the measurement point. The spatial growth rate $\tilde{\zeta}$ is obtained by performing 
(a)

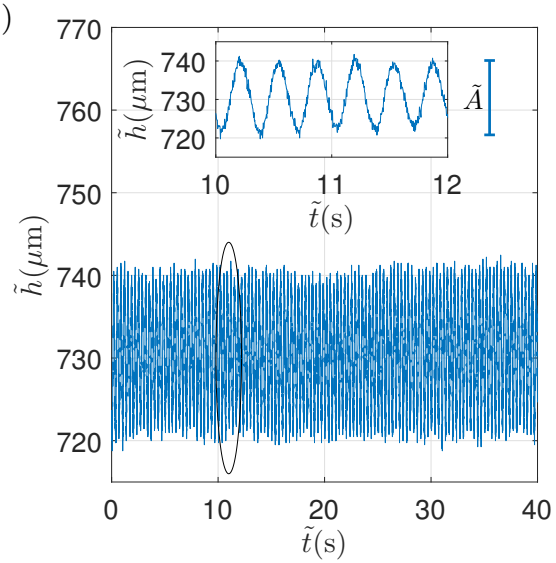

(c)

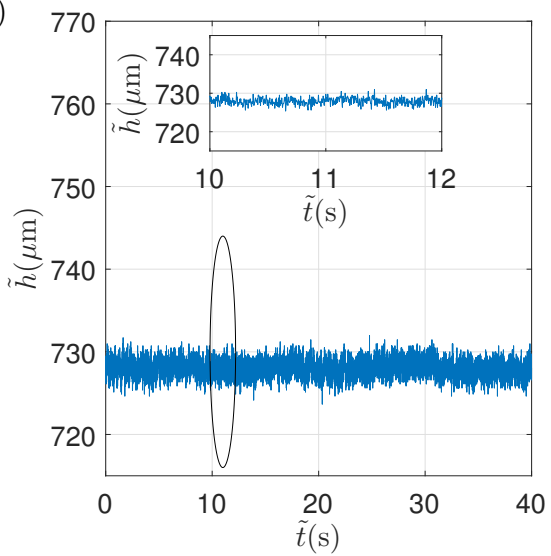

(b)

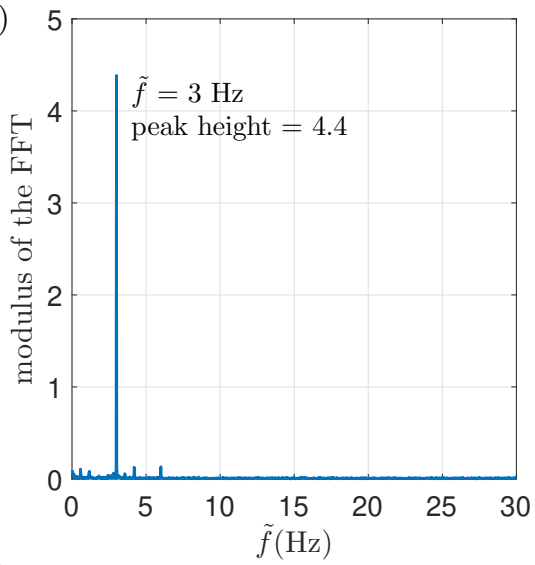

$(d)$

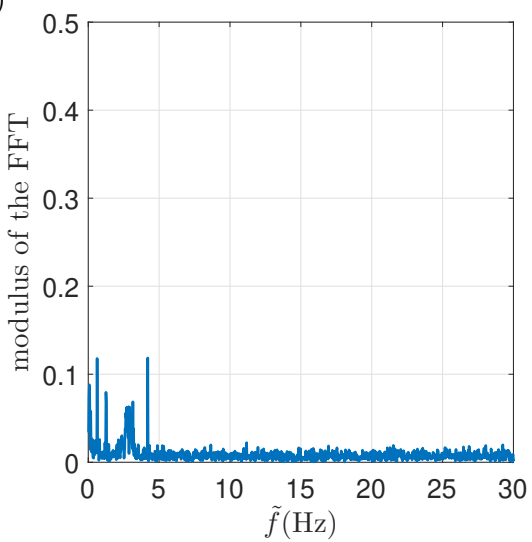

Figure 6. Examples of measurement signals with and without inlet forcing: $\beta=1.69^{\circ}$, $R e_{l}=34.8$, unconfined configuration $\tilde{H}=18 \mathrm{~mm}$. (a) Time trace of the film thickness measured by the CCI system at a fixed longitudinal location $\tilde{x}=63 \mathrm{~cm}$ and enlarged inset. Forcing frequency: $\tilde{f}=3 \mathrm{~Hz}$. (b) Corresponding modulus of the discrete Fourier transform. (c) Time evolution of the film thickness without inlet forcing. $(d)$ Corresponding modulus of the discrete Fourier transform (the scale is divided by 10 compared to panel $6 b$ ).

a linear regression on the logarithm of $\tilde{A}(\tilde{x})$. The precision of $\tilde{\zeta}$, i.e. $\Delta \tilde{\zeta}$, depends on the standard deviation of the residual of the regression, $\sigma_{\text {res }}$, and on the length $\Delta \tilde{x}$ over which the measurements are conducted: $\Delta \tilde{\zeta}=2 \sigma_{\text {res }} \Delta \tilde{x}^{-1}$. These experiments require extremely precise amplitude measurements at several positions along the channel, especially when the growth rate is weak. Figure $7 a$ shows an example of the streamwise spatial evolution of the wave amplitude. Here, $\tilde{\zeta}$ is obtained by a fitting from $\tilde{x}=10$ to $30 \mathrm{~cm}$. We observe that for $\tilde{x}>30 \mathrm{~cm}$ the spatial evolution of the wave amplitude is no longer exponential. The region of exponential growth varies depending on the experimental parameters (inclination angle, Reynolds number, forcing frequency). Accordingly, the amplitude of the inlet excitation is optimized for each experimental run so that the wave amplitude is large enough to be detected over the length of the channel while remaining in the linear regime.

The neutral stability curve $\tilde{f}_{c}\left(R e_{l}\right)$ is determined as follows. The wave amplitude is measured at two locations along the channel (amplitude $\tilde{A}_{1}$ at $\tilde{x}_{1}=54 \mathrm{~cm}$ and amplitude $\tilde{A}_{2}$ at $\tilde{x}_{2}=63 \mathrm{~cm}$ ), within the exponential growth region. Fixing $\beta$ and the forcing frequency $\tilde{f}$, we compare the two amplitudes for several Reynolds numbers to find the 
(a)

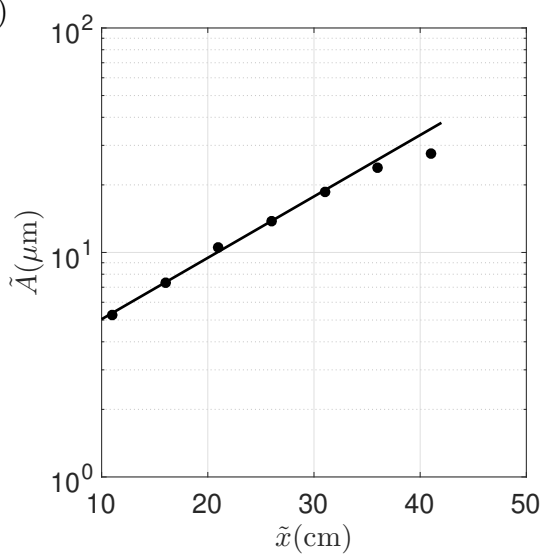

(b)

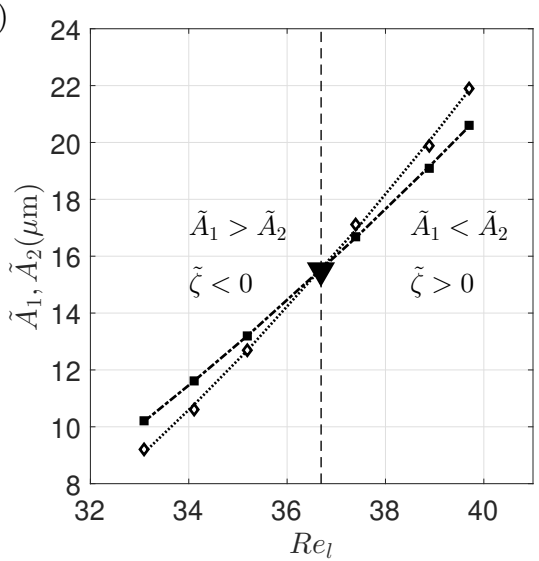

FIgURE 7. Determining the growth rate and stability threshold from experiments. $(a)$ Streamwise evolution of the wave amplitude along the channel $\left(\tilde{f}=2.6 \mathrm{~Hz}, \beta=3.05^{\circ}\right.$, $R e_{l}=34.9$, unconfined configuration $\tilde{H}=18 \mathrm{~mm}$ ). Experimental points (symbols) are fitted using an exponential curve (solid line) in the range $\tilde{x}=10-30 \mathrm{~cm}$ to obtain the spatial growth rate: $\tilde{\zeta}=6.3(1 / \mathrm{m}) \pm 0.4(1 / \mathrm{m})$. (b) Determination of the critical Reynolds number from the change in wave amplitude at two streamwise locations $\tilde{x}_{1}$ and $\tilde{x}_{2}$ when varying $\operatorname{Re}_{l}(\tilde{f}=4.2 \mathrm{~Hz}$, $\beta=1.69^{\circ}$, unconfined configuration $\left.\tilde{H}=18 \mathrm{~mm}\right)$. Filled squares: amplitude $\tilde{A}_{1}$ at $\tilde{x}_{1}=54 \mathrm{~cm}$, empty diamonds: amplitude $\tilde{A}_{2}$ at $\tilde{x}_{2}=63 \mathrm{~cm}$. The downward triangle marks the intersection, yielding the critical Reynolds number.

critical Reynolds number at which the spatial growth rate $\tilde{\zeta}$ changes sign. This threshold is determined by fitting curves to the $\tilde{A}_{i}\left(R e_{l}\right)$ data for the two locations and locating their intersection, as illustrated in figure $7 b$. The two curves in figure $7 b$ represents the wave amplitudes $\tilde{A}_{1}$ and $\tilde{A}_{2}$ measured at two different locations $\tilde{x}_{1}$ and $\tilde{x}_{2}>\tilde{x}_{1}$ as a function of $R e_{l}$. At the value of $R e_{l}$ where the two curves intersect, i.e. where $\tilde{A}_{1}=\tilde{A}_{2}$, the spatial growth rate is zero and thus $R e_{l}=R e_{c r}$. To the left of the intersection, the downstream amplitude is smaller than the upstream one $\left(\tilde{A}_{1}>\tilde{A}_{2}\right)$ and thus the film is stable. To the right of the intersection, the downstream amplitude is larger than the upstream one $\left(\tilde{A}_{1}<\tilde{A}_{2}\right)$ and thus the film is unstable. This measurement of the critical Reynolds number is performed for several forcing frequencies, thus obtaining different points on the neutral stability curve $\tilde{f}_{c}\left(R e_{l}\right)$. Subsequently, in order to cross-validate these points, the Reynolds number is fixed at the critical value corresponding to a given forcing frequency, and frequencies slightly above and below this value are scanned to verify that the film indeed changes from stable to unstable.

\section{Results}

In this section, we present our experimental and numerical linear stability results for three existing fluid combinations: (i) water in contact with air, which we mainly focus on; (ii) aqueous solution of dymethylsulfoxide (DMSO) in contact with air; (iii) aqueous solution of glycerin in contact with air. The fluid properties and the range of variation of the control parameters used for the numerical stability calculations are summarized in tables 3 and 4. 


$\begin{array}{lccccc}\text { Fluid system } & \rho_{l}\left(\mathrm{~kg} / \mathrm{m}^{3}\right) & \rho_{g}\left(\mathrm{~kg} / \mathrm{m}^{3}\right) & \nu_{l}\left(\mathrm{~m}^{2} / \mathrm{s}\right) & \nu_{g}\left(\mathrm{~m}^{2} / \mathrm{s}\right) & \gamma(\mathrm{mN} / \mathrm{m}) \\ \text { Water-air } & 1000.0 & 1.185 & 1.00 \times 10^{-6} & 15.58 \times 10^{-6} & 76.9 \\ \text { DMSO(83.11\%)-air } & 1098.3 & 1.185 & 2.85 \times 10^{-6} & 15.58 \times 10^{-6} & 48.4 \\ \text { Glycerin(50\%)-air } & 1130.0 & 1.185 & 5.02 \times 10^{-6} & 15.58 \times 10^{-6} & 69.0\end{array}$

TABLE 3. Physical properties of the fluid systems considered in the numerical stability calculations. The working liquids are: water, an aqueous solution of dymethylsulfoxide (DMSO) at $83.11 \%$ by weight, and an aqueous solution of glycerin at $50 \%$ by weight. The outer phase is air in all cases.

$\begin{array}{lcc}\text { Control parameter } & \text { Notation } & \text { Range of variation } \\ \text { Inclination angle } & \beta & 1.65^{\circ}-20^{\circ} \\ \text { Relative confinement } & \eta & 1.4-25.5 \\ \text { Liquid Reynolds number } & R e_{l} & 17-70 \\ \text { Gas Reynolds number } & R e_{g} & -250-4.8 \\ \text { Kapitza number } & K a & 331.8-3592\end{array}$

TABLE 4. Range of variation of the control parameters for the numerical stability calculations.

\subsection{Unconfined gas phase}

We start by validating our temporal linear stability calculations with our experiments in the "unconfined" configuration of figure $4 b(\tilde{H}=18 \mathrm{~mm})$. We do this comparison for two types of curves: the neutral stability curve $\tilde{f}_{c}\left(R e_{l}\right)$ and the dispersion curve of the spatial growth rate $\tilde{\zeta}(\tilde{f})$. In the latter case, we use the Gaster transformation $(2.22)$ to transform the temporal growth rate from our numerical calculations into a spatial growth rate.

Figure 8 shows the neutral stability curve $\tilde{f}_{c}\left(R e_{l}\right)$ for an inclination angle of $\beta=1.69^{\circ}$. Therein, different types of symbols, which represent measurements, indicate the stability of the investigated point: downward-pointing triangles for stable, upward-pointing triangles for unstable, and circles for neutral conditions. The solid line corresponds to the stability calculation. We point out that the precision of the inclination angle measurement using the inclinometer was not sufficient to obtain such good agreement between the two data sets. Instead, $\beta$, which is the same for all points on the neutral stability curve, was adjusted in the stability calculation until the numerical curve best matched the experimental one.

For the case of figure 8 , where the inclinometer measurement yielded $\beta=1.70^{\circ}$ with a precision of $0.05^{\circ}$, we computed numerical curves for inclination angles ranging from $1.67^{\circ}$ to $1.73^{\circ}$ with a $0.01^{\circ}$ increment. The numerical curve fitting best with the experimental data yielded an inclination angle of $\beta=1.69^{\circ}$. When varying $\beta$, not only the numerical curve changes but also the experimental points, because the flow rate is obtained from equation (3.1), where $K_{l}$ depends on $\beta$. To demonstrate the sensitivity of the comparison in figure 8 w.r.t. to the inclination angle $\beta$, we have included two additional figures in appendix $\mathrm{B}$, showing the comparison for $\beta=1.68^{\circ}$ and $\beta=1.70^{\circ}$.

Figure 9 compares numerical and experimental data for a larger inclination angle. Panel $9 a$ represents the neutral stability curve for $\beta=3^{\circ}$, and panel $9 b$ the dispersion curve of the spatial growth rate for $\beta=3.08^{\circ}$ and $R e_{l}=31$. As experimental data were not acquired on the same day, the precise value of $\beta$ is slightly different for the two 


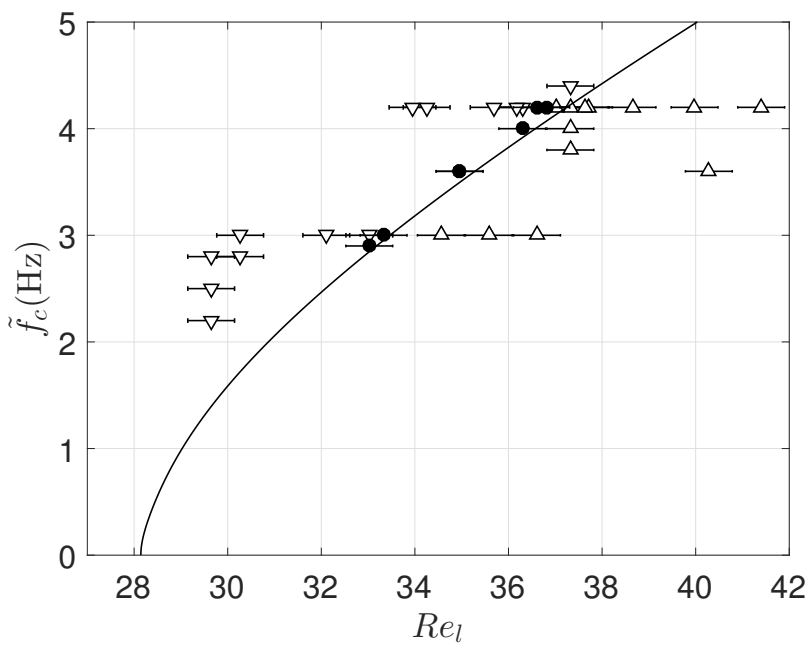

Figure 8. Comparison of numerical (line) and experimental (symbols) stability results at $\beta=1.69^{\circ}$ in the "unconfined" configuration (figure $4 b$ ): neutral stability curve. Symbols indicate the stability of the investigated point: downward-pointing triangles for stable, upward-pointing triangles for unstable, and circles for neutral conditions.

$(a)$

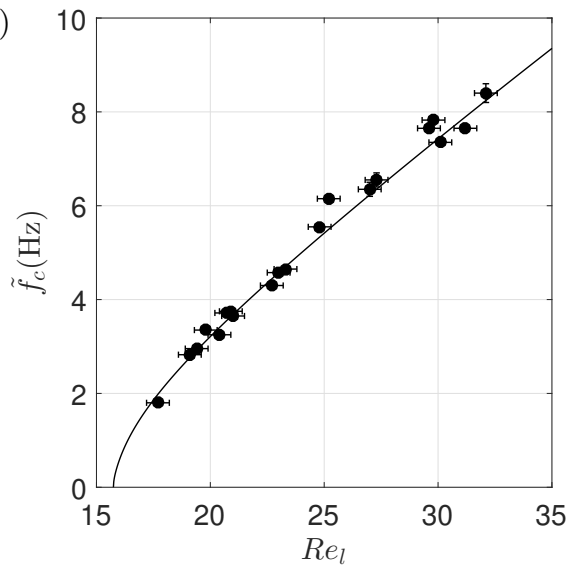

(b)

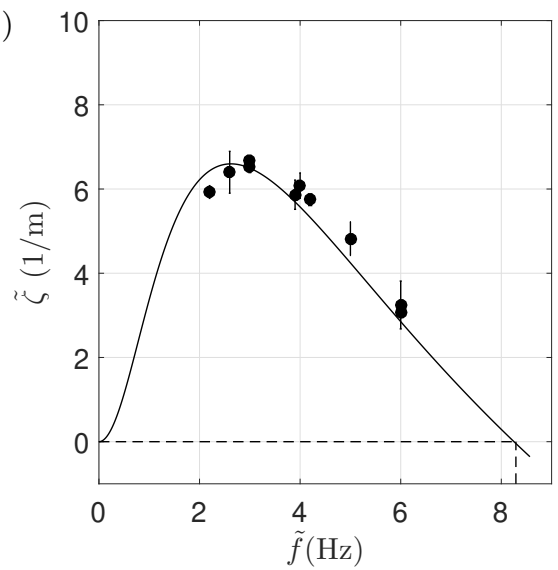

Figure 9. Comparison of numerical (solid line) and experimental (filled circles) stability results: unconfined configuration. (a) Neutral stability curve for $\beta=3^{\circ} ;(b)$ dispersion curve of the spatial growth rate for $\beta=3.08^{\circ}$ and $R e_{l}=31$. The precise value of $\beta$ is slightly different for the two panels as experimental data were obtained from experimental runs on different days.

panels. Agreement between the experimental and numerical dispersion curves confirms that the Gaster transformation is applicable here (see also Brevdo et al. (1999)).

\subsection{Confined gas phase: aerostatic pressure difference}

We now study how the stability of the falling liquid film changes when the gas phase is significantly confined. To quantify the degree of confinement, we use the ratio $\eta(2.7)$ relating the gap height $H$ and the thickness of the primary film flow $h_{0}$. To quantify the effect of $\eta$ on film stability, we will compare our stability results with the limiting case of a passive gas $\left(\Pi_{\mu}, \Pi_{\rho} \rightarrow 0\right)$, where the confinement plays no role. This limit will be denoted with the superscript $\infty$.

We first study the situation where the gas is subject to an aerostatic pressure gradient. 


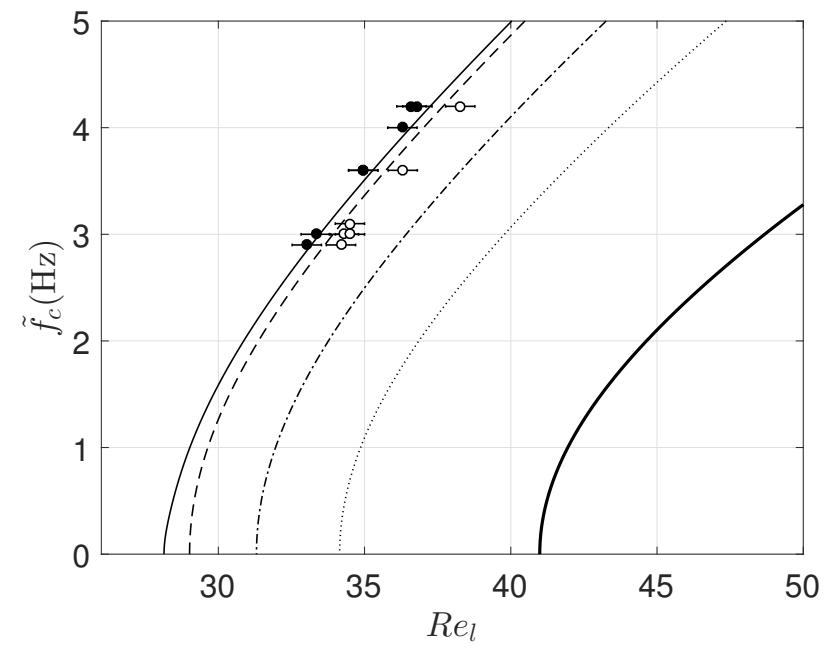

Figure 10. Neutral stability curves of a water film $(K a=3592)$ inclined at $\beta=1.69^{\circ}$ in contact with aerostatic air at different confinement levels $\eta_{c r}=\eta\left(\alpha_{c}=0\right)$. Filled symbols: "unconfined" experiment with $\eta_{c r}=25.5$ (panel $4 b, \tilde{H}=18 \mathrm{~mm}$ ); open symbols: confined experiment with $\eta_{c r}=7.54$ (panel $\left.4 a, \tilde{H}=5.1 \mathrm{~mm}\right)$. Stability calculations are represented with lines. Thin solid: "unconfined" $\left(\eta_{c r}=25.5, \tilde{H}=18 \mathrm{~mm}\right)$; dashed line: confined at $\tilde{H}=5.1 \mathrm{~mm}\left(\eta_{c r}=7.54\right)$; dash-dotted line: $\tilde{H}=2.5 \mathrm{~mm}\left(\eta_{c r}=3.60\right)$; dotted line: $\tilde{H}=2 \mathrm{~mm}\left(\eta_{c r}=2.80\right)$; thick solid line: $\tilde{H}=1.7 \mathrm{~mm}\left(\eta_{c r}=2.23\right)$.

In that case, the gas moves only as a reaction to the film's motion. We start by validating our temporal stability calculations with our own experiments in the confined configuration $\tilde{H}=5.1 \mathrm{~mm}$ (panel $4 a$ ) and the "unconfined" configuration $\tilde{H}=18 \mathrm{~mm}$ (panel $4 b$ ) at an inclination angle $\beta=1.69^{\circ}$, using water as working liquid.

The two leftmost curves in figure 10 represent the calculated neutral stability curves $\tilde{f}_{c}\left(R e_{l}\right)$ for these two configurations, while symbols represent the corresponding experimental data. Both data sets evidence a clear stabilization of the falling film due to increased confinement of the gas phase (dashed curve, open circles), and their agreement is gratifying.

The dimensional gap height $\tilde{H}$ along the curves in figure 10 is constant, meaning that the relative confinement $\eta$ changes, as $\widetilde{h}_{0}$ changes with $R e_{l}$. Nonetheless, a representative value for $\eta$ can be given for each curve by evaluating it at the critical Reynolds number $R e_{c r}$ (where the curve intersects the $x$-axis), i.e. $\eta_{c r}=\eta\left(\alpha_{c}=0\right), \alpha_{c}$ being the cut-off wavenumber. The quantity $\eta_{c r}$ is quite large for the two leftmost curves in figure 10 $\left(\eta_{c r}>7\right)$, and this explains why the observed confinement-induced stabilization is rather weak (experimental wave amplitudes are reported in figure 24 of appendix C). However, when decreasing $\eta_{c r}$, the confinement-induced stabilization becomes much stronger, as evidenced by the three additional curves in figure 10 obtained from our stability calculations. For example, at $\eta_{c r}=2.23$ (thick solid line), the critical Reynolds number number $R e_{c r}$, below which the film is always stable, is increased by over $50 \%$ compared to the "unconfined" configuration (thin solid line).

This means that the confinement can fully stabilize the Kapitza instability at a given Reynolds number. We now investigate this suppression of the instability in further detail based on our stability calculations. In figure $11 a$, we have plotted the temporal growth rate for a water film at $R e_{l}=23.9$ and $\beta=3^{\circ}$ as a function of the wavenumber $\alpha$ for different values of $\eta$ (panel $11 b$ represents the corresponding wave celerity $c_{r}$ ). We see 
$(a)$

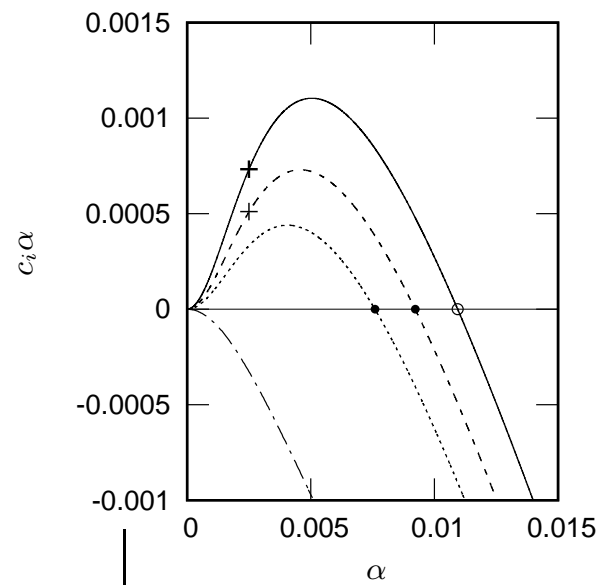

$(b)$

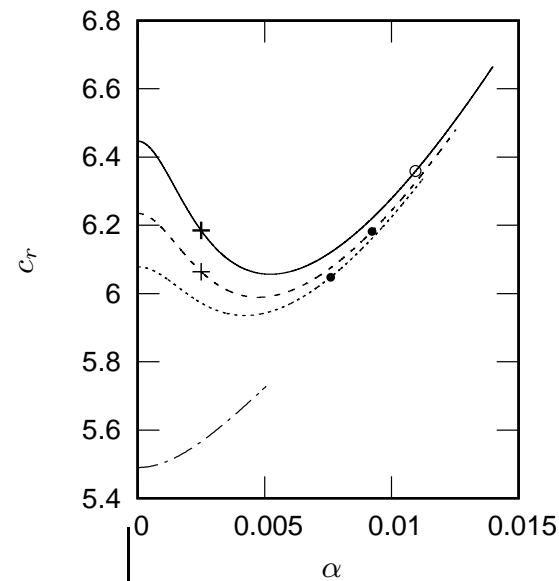

Figure 11. Stability calculations for a water film $(K a=3592)$ at $R e_{l}=23.9$ and $\beta=3^{\circ}$ in contact with aerostatic air. Effect of confinement on $(a)$ temporal growth rate and $(b)$ wave celerity. Solid line: passive-gas limit; dashed line: $\eta=3.6$; dotted line: $\eta=2.8$; dash-dotted line: $\eta=2$. The + symbol marks data points that will be discussed in $\S 5$. The filled and open circles correspond to the cut-off wavenumber $\alpha_{c}$ for the confined and passive-gas configurations.

$(a)$

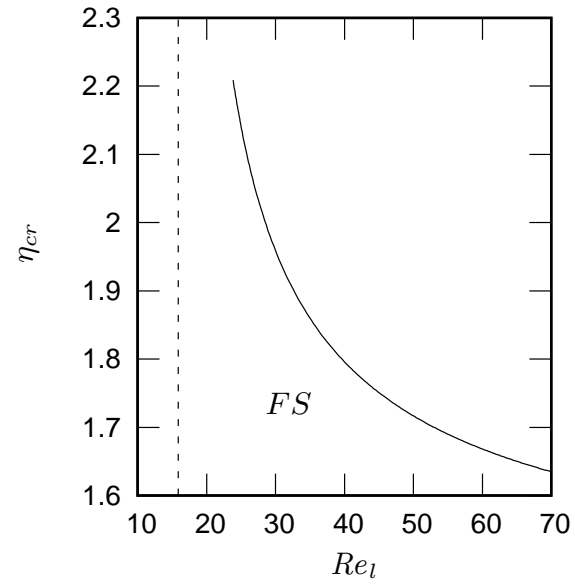

(b)

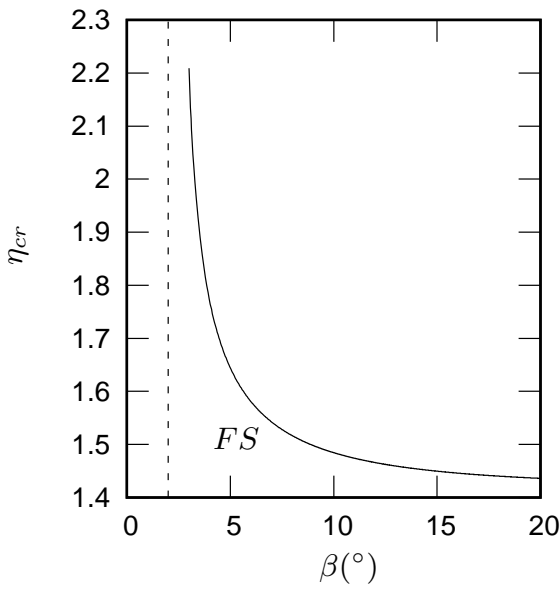

FiguRE 12. Numerical calculation of the critical confinement $\eta_{c r}=\eta\left(\alpha_{c}=0\right)$ below which a water film $(K a=3592)$ in contact with aerostatic air is fully stabilized $(F S)$. $(a)$ Effect of the liquid Reynolds number $R e_{l}$ at $\beta=3^{\circ}$. The vertical dashed line marks the critical Reynolds number in the passive-gas limit $R e_{c r}^{\infty}=5 / 6 \cot \beta=15.9$; $(b)$ effect of the inclination angle $\beta$ at $R e_{l}=23.9$. The vertical dashed line marks the critical angle in the passive-gas limit $\beta_{c r}^{\infty}=\arctan \left(5 / 6 / R e_{l}\right)=2^{\circ}$.

that the unstable range, i.e. where the wavenumber $\alpha$ is smaller than the cut-off value $\alpha_{c}$ (intersection of the curves with $c_{i} \alpha=0$ ) decreases with $\eta$ and entirely disappears at $\eta=2$. At this point, the Kapitza instability has been fully suppressed.

The critical relative confinement $\eta_{c r}$ for the suppression of the instability is plotted in figure 12 against the liquid Reynolds number $R e_{l}$ (panel 12a) and the inclination angle $\beta$ (panel 12b), respectively. We see that it is easier to suppress the falling film instability the smaller $\operatorname{Re}_{l}$ and $\beta$.

No such curve can be obtained for the remaining control parameter, the Kapitza number $K a$, as the onset of the long-wave instability is unaffected by surface tension. 
(a)

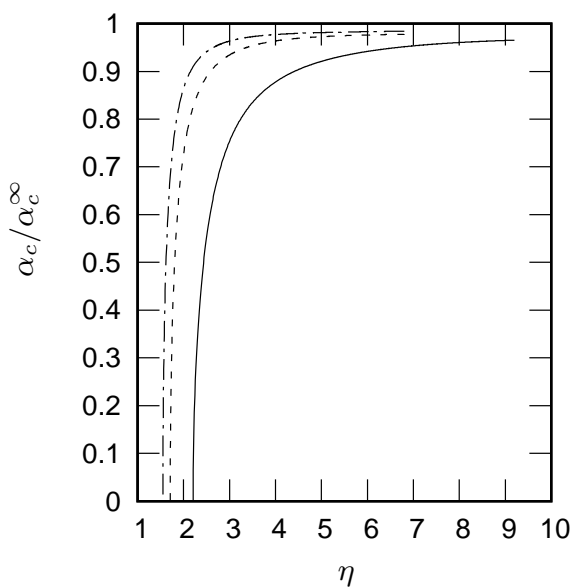

(b)

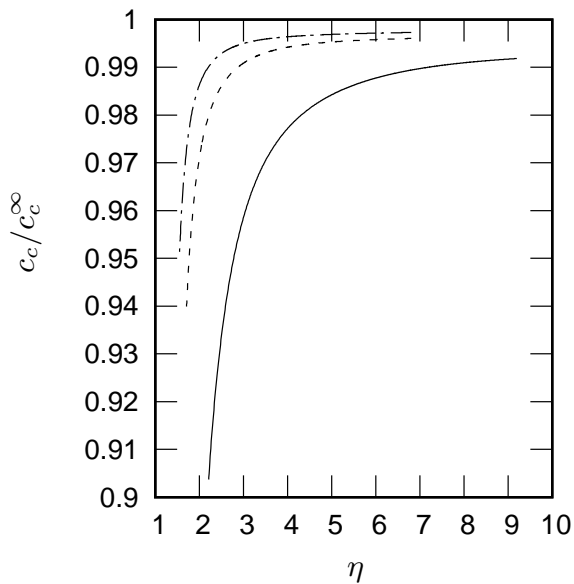

Figure 13. Stabilization due to confinement for different working liquids at $R e_{l}=23.9$ and $\beta=3^{\circ}$ in contact with aerostatic air. (a) Cut-off wavenumber and (b) cut-off wave celerity compared to their passive-gas limits $\alpha_{c}^{\infty}$ and $c_{c}^{\infty}$ (open circles in panels $11 a$ and $11 b$ ). Solid line: water $(K a=3592)$; dashed line: aqueous solution of dymethylsulfoxide at $83.11 \%$ by weight $(K a=509.5)$; dash-dotted line: aqueous solution of glycerin at $50 \%$ by weight $(K a=331.8)$.

Instead, we have plotted in figure 13 the cut-off wavenumber (panel 13a) and the corresponding wave celerity (panel $13 b$ ) as a function of $\eta$ for the three studied liquids, which display a substantial variation of $K a$. In both panels, the plotted quantity is related to its value in the limit of a passive gas. We see that the stabilizing effect of confinement is felt earlier for water $(K a=3592)$ compared to aqueous solutions of dymethylsulfoxide $(K a=509.5)$ and glycerol $(K a=331.8)$. Panel $13 b$ shows that the confinement-induced stabilization of the liquid film is accompanied by a decrease of the wave celerity.

\subsection{Confined gas phase: counter-current flow}

We now investigate how confinement affects the stability of the falling liquid film when it is sheared by a counter-current gas flow. For this, we apply an adverse pressure difference $\Delta p>0$, which is larger than the aerostatic pressure difference $\Delta p^{a}=\Lambda \sin \beta$. The strength of the gas flow is quantified by the pressure difference ratio $M=\Delta p / \Delta p^{a}$.

Figure 14 represents stability calculations for a falling water film at $\beta=3^{\circ}$ and $R e_{l}=$ 23.9. The relative confinement is fixed to $\eta=3.6$ and the adverse pressure difference is increased. Panel $14 a$ shows that increasing the counter-current gas flow increasingly stabilizes the film until the point of fully suppressing the Kapitza instability (dash-dotteddotted line). Panel $14 b$ shows that this stabilization is accompanied by a decrease in wave celerity.

The level of stabilization strongly depends on the inclination angle $\beta$, as shown in panels $15 a$ and $15 b$, which represent contours of the cut-off wavenumber (for $R e_{l}=23.9$ ) and critical Reynolds number related to their aerostatic limit as a function of $\beta$ and $M$. At low inclination angles and relatively high adverse pressure gradients, the cut-off wavenumber is reduced by up to $70 \%$ with respect to the aerostatic case and the critical Reynolds number is increased by up to $40 \%$.

The dispersion curves in figure 14 exhibit a number of features that seem to contradict the stability results of Alekseenko et al. (2009), Vellingiri et al. (2015), and Trifonov (2017), which were obtained for a weakly-confined turbulent gas flow $(\eta=24-260$ in Trifonov (2017)). Firstly, increasing the gas flow, monotonically reduces our unstable range of wavenumbers, whereas Vellingiri et al. (2015) and Trifonov (2017) found that the 
(a)

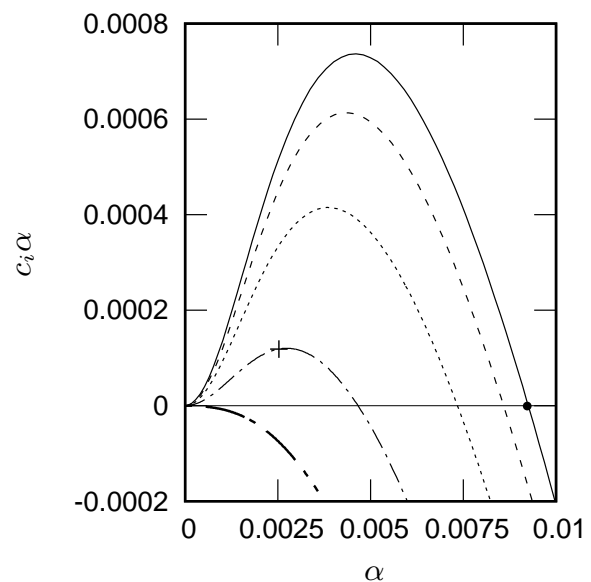

$(b)$

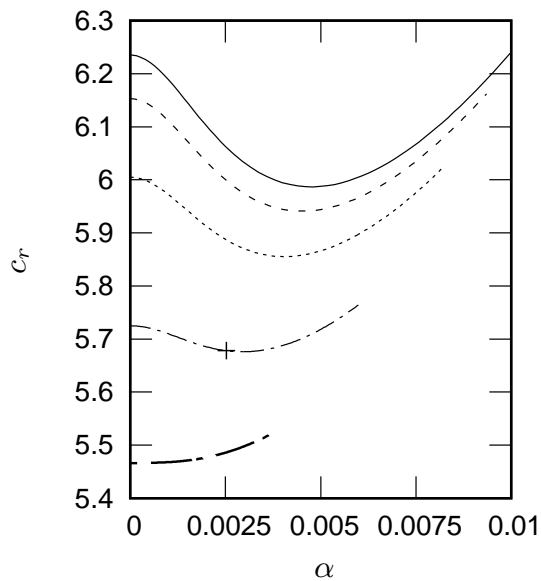

FIGURE 14. Influence of an increasingly strong counter-current air flow on the stability of a falling water film $(K a=3592)$ at $\beta=3^{\circ}, R e_{l}=23.9$ and $\eta=3.6$. (a) Temporal growth rate; (b) wave celerity. Solid line: $M=1\left(R e_{g}=3.0\right)$; dashed line: $M=10\left(R e_{g}=-0.94\right)$; dotted line: $M=25\left(R e_{g}=-7.2\right)$; dash-dotted line: $M=50\left(R e_{g}=-17.1\right)$; thick dash-dotted line: $M=70\left(R e_{g}=-24.4\right)$. The + symbol marks data points that will be discussed in $\S 5$. The filled circle in $(a)$ marks the cut-off wavenumber $\alpha_{c}^{a}$ for the aerostatic configuration.

cut-off wavenumber can first decrease and then increase with the gas velocity magnitude. Secondly, our growth rate is reduced at all wavenumbers, whereas Alekseenko et al. (2009) found that increasing the gas velocity magnitude may reduce the cut-off wavenumber but increase the maximal growth rate at the same time. In that case, long waves are amplified and short waves are stabilized.

It turns out that these differences are not due to the laminar gas flow conditions, but to the strong confinement levels studied here. Indeed, for weaker confinement levels, we recover the same qualitative behaviour as Alekseenko et al. (2009), Vellingiri et al. (2015), and Trifonov (2017), notwithstanding that the gas flow is laminar in our case. This is shown in figures 16 , where we consider a significantly weaker confinement $(\eta \geqslant 7)$. Figure $16 a$ shows that the cut-off wavenumber (filled circles) now varies non-monotonically with increasing gas flow. Interestingly, this curve displays an additional local maximum in addition to the local minimum reported by Vellingiri et al. (2015) and Trifonov (2017). In the same graph, we have also plotted the maximally-amplified wavenumber (filled triangles), which varies in the same manner. Panel $16 b$ shows the corresponding curve for the maximum growth rate, which displays only a local minimum.

In panel $16 c$, we have plotted growth rate dispersion curves for three representative points from panel 16a. In contrast to the dispersion curves in panel $14 a$ (where $\eta=3.6$ ), we see that increasing the gas flow can now stabilize short waves while amplifying long waves (compare solid and dot-dashed lines), in accordance with Alekseenko et al. (2009). For an even weaker confinement level $(\eta=9)$, we find that an increased gas flow can destabilize the film at all wavenumbers, as shown in figure $17 a$. Panel $17 b$, which plots different characteristic points of the growth rate dispersion curve as a function of $\eta$, allows to identify the critical confinement at which this transition occurs $\left(\eta=8\right.$ for $R e_{l}=17$ and $\left.\beta=3^{\circ}\right)$. Just before the transition $(\eta=7.5-8)$, the cut-off wavenumber is reduced w.r.t. the aerostatic limit while the maximum growth rate is increased, similarly to what was observed by Alekseenko et al. (2009). 
(a)

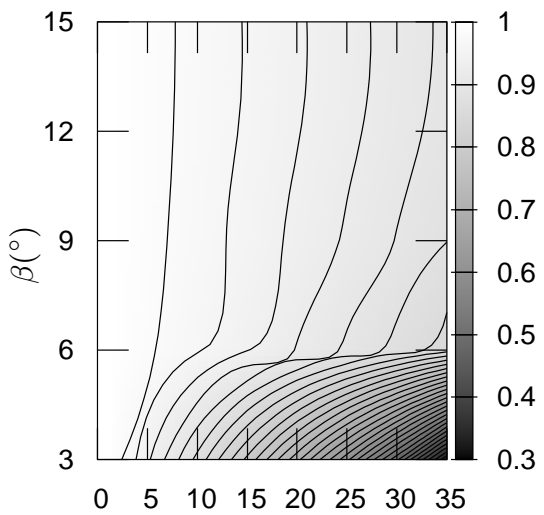

$M$ (b)

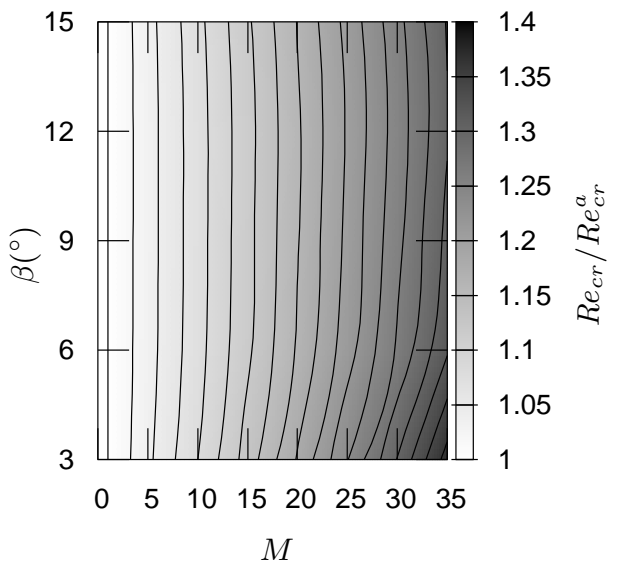

FIGURE 15. Effect of an increasingly strong counter-current air flow $\left(M=\Delta p / \Delta p^{a}\right)$ on the stability of a falling water film $(K a=3592)$ at $\beta=3^{\circ}$ and $\eta=2.8$. (a) Deviation of the cut-off wavenumber $\alpha_{c}$ from its aerostatic limit $\alpha_{c}^{a}$ at $R e_{l}=23.9 ;(b)$ deviation of the critical Reynolds number $R e_{c r}$ from its aerostatic limit $R e_{c r}^{a}$. Darker regions correspond to a greater stabilization.

(a)
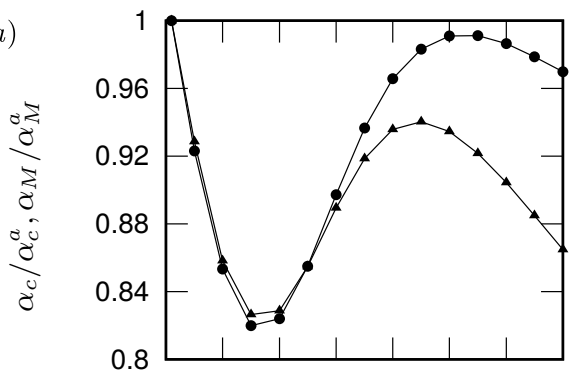

(b)

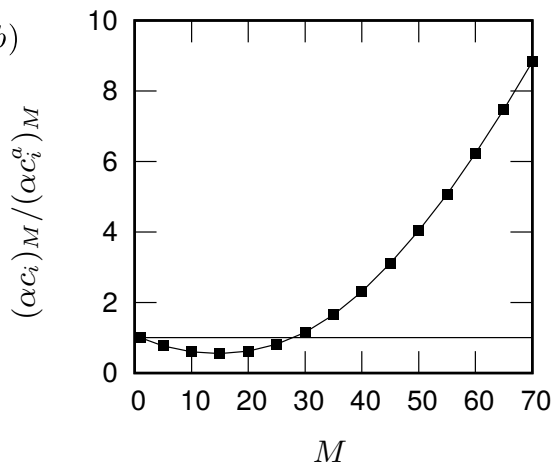

$(c)$

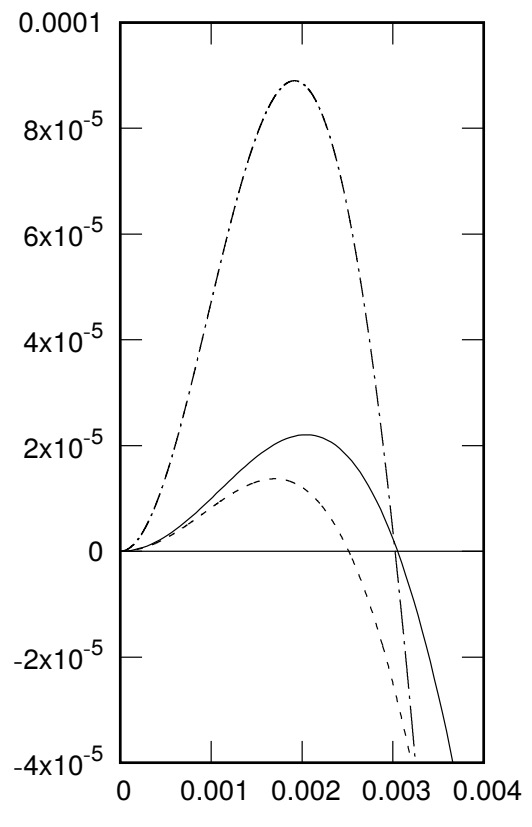

FiguRE 16. Effect of an increasingly strong counter-current air flow $\left(M=\Delta p / \Delta p^{a}\right)$ on the stability of a falling water film $(K a=3592)$ at $\beta=3^{\circ}, \eta=7$ and $R e_{l}=17$. (a) Deviation of the cut-off wavenumber $\alpha_{c}$ (filled circles) and most unstable wavenumber $\alpha_{M}$ (filled triangles) from their respective aerostatic limits (superscript $a$ ); $(b)$ deviation of the maximum growth rate $\left(\alpha c_{i}\right)_{M}$ from the corresponding aerostatic limit $\left(\alpha c_{i}^{a}\right)_{M}$. The horizontal solid line denotes $\left(\alpha c_{i}\right)_{M}=\left(\alpha c_{i}^{a}\right)_{M} ;(c)$ temporal growth rate. Solid line: $M=1\left(R e_{g}=5.0\right)$; dashed line: $M=20$ $\left(R e_{g}=-67.5\right)$; dash-dotted line: $M=50\left(R e_{g}=-175\right)$.

\section{Explanation of the stabilization mechanism}

To identify the mechanism responsible for the confinement-induced stabilization of the

Kapitza instability, we focus on three representative cases for which linear stability data 
$(a)$

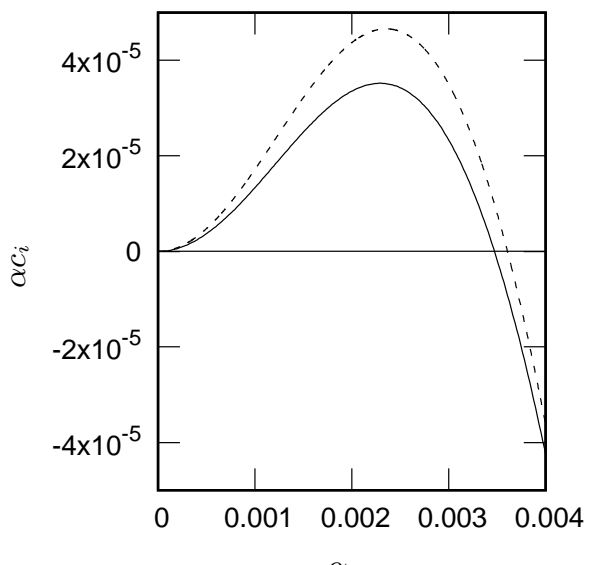

(b)

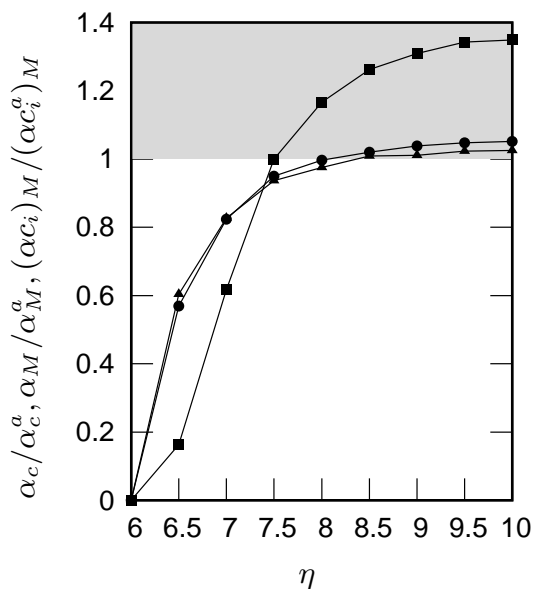

Figure 17. Effect of the relative confinement $\eta$ on the stability of a falling water film $(K a=3592)$ at $\beta=3^{\circ}, R e_{l}=17$ and $R e_{g}=-67.5$. (a) temporal growth rate. Solid line: $M=1$ and $\eta=9$; dashed line: $R e_{g}=-67.5(M=9.1)$ and $\eta=9 ;(b)$ deviation of the cut-off wavenumber $\alpha_{c}$ (filled circles), most unstable wavenumber $\alpha_{M}$ (filled triangles) and maximum growth rate $\left(\alpha c_{i}\right)_{M}$ (filled squares) from their respective aerostatic limits. The grey area marks the unstable region compared to the aerostatic scenario.

were reported in $\S 4$ (the two points marked by plus symbols in figure 11 and the single point also marked by a plus symbol in figure 14). In all three cases, we have $R e_{l}=23.9$ and $\beta=3^{\circ}$, whereas the conditions for the gas differ as follows: (i) aerostatic gas with weak relative confinement $\eta=12.4$; (ii) aerostatic gas with strong relative confinement $\eta=3.6$; (iii) counter-current gas with $R e_{g}=-17.1$ and strong relative confinement $\eta=3.6$.

For each case, we evaluate the linear perturbation of different interfacial quantities based on a representative unstable mode fixed by the wavenumber $\alpha=0.0025$ (situated on the ascending branch of the growth rate curves in figures 11 and 14). These perturbations can all be expressed in terms of the streamfunction perturbation amplitudes $\hat{\phi}$ and $\hat{\psi}(2.14)$, which are the eigenfunctions of the Orr-Sommerfeld problem (2.15). For example, the film thickness perturbation $h^{\star}(2.20)$ can be written as:

$$
\begin{gathered}
h^{\star}=\hat{h} \exp \left[i \alpha(x-c t)+i \Theta_{h}\right], \\
\hat{h}=\hat{\phi} \sqrt{\operatorname{Re}\{1 / \underline{c}\}^{2}+\operatorname{Im}\{1 / \underline{c}\}^{2}}, \quad \Theta_{h}=\arctan [\operatorname{Im}\{1 / \underline{c}\} / \operatorname{Re}\{1 / \underline{c}\}],
\end{gathered}
$$

where $\Theta_{h}$ designates the phase shift with respect to the stream-function perturbation $(2.14 a)$. Further, we are interested in the perturbed adverse tangential shear stress $\tau_{t}$ exerted by the gas on the film surface:

$$
\tau_{t}=T_{t}+\tau_{t}^{\star}
$$

where $T_{t}<0$ is the tangential interfacial shear stress of the primary flow and the shear stress perturbation $\tau_{t}^{\star}$ subsumes the two contributions associated with interfacial 
505

quantities defined by the last two terms in (2.18):

$$
\begin{gathered}
\tau_{t}^{\star}=\hat{\tau}_{t} \exp \left[i \alpha(x-c t)+i \Theta_{\tau}\right], \\
\hat{\tau}_{t}=\sqrt{\operatorname{Re}\left\{Z_{\tau}\right\}^{2}+\operatorname{Im}\left\{Z_{\tau}\right\}^{2}}, \quad \Theta_{\tau}=\arctan \left[\operatorname{Im}\left\{Z_{\tau}\right\} / \operatorname{Re}\left\{Z_{\tau}\right\}\right], \\
Z_{\tau}=\Pi_{\rho}{ }^{-1}\left[\hat{\phi}^{\prime \prime}+U_{l}^{\prime \prime} \hat{\phi} \underline{c}^{-1}+\alpha^{2} \hat{\phi}\right],
\end{gathered}
$$

where the purely real amplitude $\hat{\tau}_{t}$ of the perturbation and its phase shift $\Theta_{\tau}$ follow from the complex amplitude $Z_{\tau}$. Similarly, and with the help of $(2.21 b)$, we obtain for the perturbed interfacial gas pressure $p_{g}^{\star}$ :

$$
\begin{gathered}
p_{g}^{\star}=\hat{p}_{g} \exp \left[i \alpha(x-c t)+i \Theta_{p}\right], \\
\hat{p}_{g}=\sqrt{\operatorname{Re}\left\{Z_{p}\right\}^{2}+\operatorname{Im}\left\{Z_{p}\right\}^{2}}, \quad \Theta_{p}=\arctan \left[\operatorname{Im}\left\{Z_{p}\right\} / \operatorname{Re}\left\{Z_{p}\right\}\right], \\
Z_{p}=\underbrace{c \hat{\psi}^{\prime}+U_{g}^{\prime} \hat{\psi}}_{Z_{p}^{\rho}}+\underbrace{\Pi_{\mu}\left(i \alpha \Pi_{\rho}\right)^{-1}\left(\hat{\psi}^{\prime \prime \prime}-\alpha^{2} \hat{\psi}^{\prime}\right)}_{Z_{p}^{\mu}}-\underbrace{\cos \beta \hat{\phi} \underline{c}^{-1}}_{Z_{p}^{\beta}},
\end{gathered}
$$

where the components $Z_{p}^{\rho}$ and $Z_{p}^{\mu}$ of the complex amplitude $Z_{p}$, through $(2.21 b)$, can be traced directly to the inertial and viscous contributions in the streamwise momentum equation, whereas $Z_{p}^{\beta}$ comes from the last term in (2.18).

Figures $18 b$ and $18 c$ represent the perturbation profiles of the shear stress $(5.3)$ and pressure (5.4) for the three studied cases, allowing to discern their phase shifts $\Theta_{\tau}-\Theta_{h}$ and $\Theta_{p}-\Theta_{h}$ with respect to the film thickness perturbation profile (panel 18a). These profiles have been scaled differently for the three cases, in order to facilitate a comparison within the same graph. The respective perturbation amplitudes $\hat{\tau}_{t}$ and $\hat{p}_{g}$ and that of the gas-side normal viscous stress $\hat{\tau}_{n}$ are given in table 5 . In addition, table 6 compares the weight of the inertial and viscous contributions to the pressure perturbation's phase shift $\Theta_{p}$ via the arguments $\operatorname{Im}\left\{Z_{p}^{\rho}\right\} / \operatorname{Re}\left\{Z_{p}\right\}$ and $\operatorname{Im}\left\{Z_{p}^{\mu}\right\} / \operatorname{Re}\left\{Z_{p}\right\}$.

From these data, we may conclude: (i) the perturbation of the normal viscous stress is negligible compared to that of the pressure; (ii) the perturbation amplitudes $\hat{\tau}_{t}$ and $\hat{p}_{g}$ increase both with confinement and gas velocity; (iii) for strong confinement, the phase shift with respect to the film thickness perturbation is fixed to $\Theta_{\tau}-\Theta_{h} \approx \pi$ for the shear stress perturbation and $\Theta_{p}-\Theta_{h} \approx \pi / 2$ for the pressure perturbation, and this does not change significantly when the gas velocity is increased; (iv) for strong confinement, the phase shift $\Theta_{p}$ is entirely governed by viscous effects and inertia plays no role.

The $\Theta_{p}-\Theta_{h} \approx \pi / 2$ phase shift of the pressure perturbation means that its second derivative $\partial_{x x} p_{g}^{\star}$ vanishes at the film thickness extrema. As a result, its effect on stability is neutral, i.e. it neither reinforces nor attenuates the film thickness perturbation. This phase shift follows directly from the dominance of viscous effects in the gas flow, i.e. $\operatorname{Im}\left\{Z_{p}^{\mu}\right\} \gg \operatorname{Im}\left\{Z_{p}^{\rho}\right\}$. Indeed, in this inertialess limit, the pressure gradient is proportional to one over the third power of the local gas layer thickness (locally developed Poiseuille-Couette flow). Conversely, in the case of inertia-dominated flow, the pressure would be proportional to the square of the local gas velocity (following the Bernoulli equation) and thus its minimum would arise at the maximum of the film thickness perturbation, giving an anti-cyclic $\left(\Theta_{p}-\Theta_{h}=\pi\right)$ phase shift. This would have a destabilizing effect. However, for our case of strongly-confined gas flow, $\Theta_{p}-\Theta_{h} \approx \pi / 2$ and the pressure perturbation plays no role in determining the state of stability. This is in agreement with Trifonov (2017), who, in his stability analysis, did not find any 


$\begin{array}{rcrccc}\eta & \tilde{H}(\mathrm{~mm}) & M & \hat{\tau}_{t} / \hat{\tau}_{t}^{(\eta=12.4)} & \hat{p}_{g} / \hat{p}_{g}^{(\eta=12.4)} & \hat{\tau}_{n} / \hat{p}_{g} \\ 12.4 & 6.43 & 1 & 1.0 & 1.0 & 0.09227 \\ 3.6 & 1.87 & 1 & 5.30231 & 17.2737 & 0.00531 \\ 3.6 & 1.98 & 50 & 12.5435 & 61.1488 & 0.04583\end{array}$

TABLE 5. Ratio between the perturbation amplitudes (computed through (2.18)) of the stresses exerted by an air flow on a water film inclined at $\beta=3^{\circ}$ with $R e_{l}=23.9$ : tangential shear stress $\hat{\tau}_{t}$, static pressure $\hat{p}_{g}$ and normal viscous stress $\hat{\tau}_{n}$. Wavenumber: $\alpha=0.0025$.

\begin{tabular}{rcrccc}
\multicolumn{1}{c}{$\eta$} & $\tilde{H}(\mathrm{~mm})$ & $M$ & $\operatorname{Im}\left\{Z_{p}^{\rho}\right\} / \operatorname{Re}\left\{Z_{p}\right\}$ & $\operatorname{Im}\left\{Z_{p}^{\mu}\right\} / \operatorname{Re}\left\{Z_{p}\right\}$ & $\operatorname{Im}\left\{Z_{p}^{\beta}\right\} / \operatorname{Re}\left\{Z_{p}\right\}$ \\
12.4 & 6.43 & 1 & 0.08325 & 0.99695 & 0.05462 \\
3.6 & 1.87 & 1 & 0.04240 & 14.7428 & 0.02596 \\
3.6 & 1.98 & 50 & 0.00324 & 7.68910 & 0.00021
\end{tabular}

TABLE 6. Different contribution to the pressure perturbation's phase shift according to (5.4). Flow conditions as in table 5.

unstable mode related to the Kelvin-Helmholtz instability for flow conditions similar to those studied here.

Thus, it is the perturbation of the interfacial tangential shear stress that is responsible for the confinement-related stabilization of the falling liquid film, as suggested by Tilley et al. (1994). In particular, this results from the $\Theta_{\tau}-\Theta_{h} \approx \pi$ phase shift of the shear stress perturbation with respect to the film thickness perturbation $h^{\star}$, which remains virtually unchanged over a wide range of confinement levels and gas velocities (see panels $19 a$ and $19 b)$. Due to this, the adverse tangential shear stress $\tau_{t}(5.2)$ is more negative at the wave hump and less negative at the wave trough. This is principally caused by the change in cross section for the gas flow, which is smaller above the wave hump and greater above the wave trough.

So, how does the profile of the tangential stress perturbation $\tau_{t}^{\star}$ in figure $18 b$ stabilize the film? First, we recall that the driving mechanism of the Kapitza instability is inertia, which causes the liquid flow rate $q_{l}(x, t)$ to lag behind its inertialess target value:

$$
\mathrm{q}(x, t)=-K_{l} \frac{h^{3}}{3}+\tau_{t} \frac{h^{2}}{2},
$$

where $K_{l}$ is defined in $(2.9 a)$. Just upstream of the wave hump, where q decreases in time as the wave passes by, the actual flow rate $q_{l}$ is slightly too high, while it is slightly too low just downstream of the hump, where $\mathrm{q}$ increases in time. The resulting discrepancy in flow into and out of the hump causes it to grow. The effect is stronger the more the actual flow rate $q_{l}$ lags behind its target value $\mathrm{q}$, and that depends on how steeply $\mathrm{q}$ changes within the wavy film.

According to (5.5), the effect of gravity (through the $h^{3}$ term in (5.5)) tends to increase variations in q between the wave hump and wave trough as a result of the change in film thickness $h$. The adverse tangential interfacial stress $\tau_{t}$, through its perturbation $\tau_{t}^{\star}$ (figure $18 b$ ), counter-acts this effect. Indeed, $\tau_{t}^{\star}$ acts to homogenize the flow rate within the film, slowing the flow in the wave hump to a greater extent than underneath the wave trough. This is a direct result of the phase lag observed between the film thickness and the shear stress perturbation (figure 18), and the effect increases with the relevance of the second term in (5.5). 
(a)

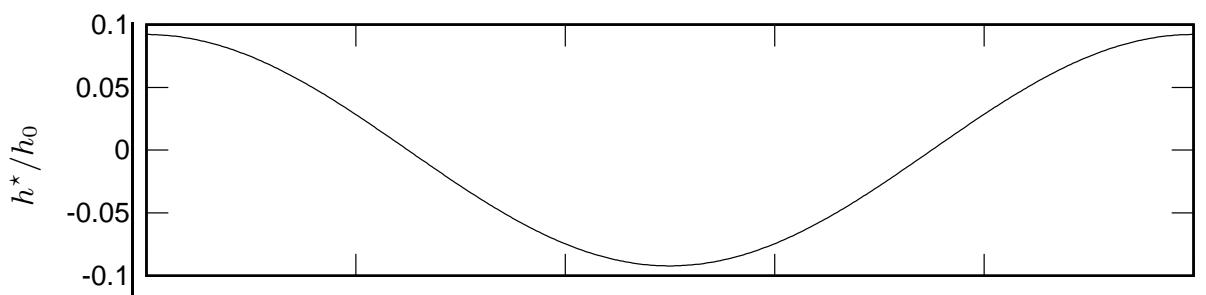

(b)

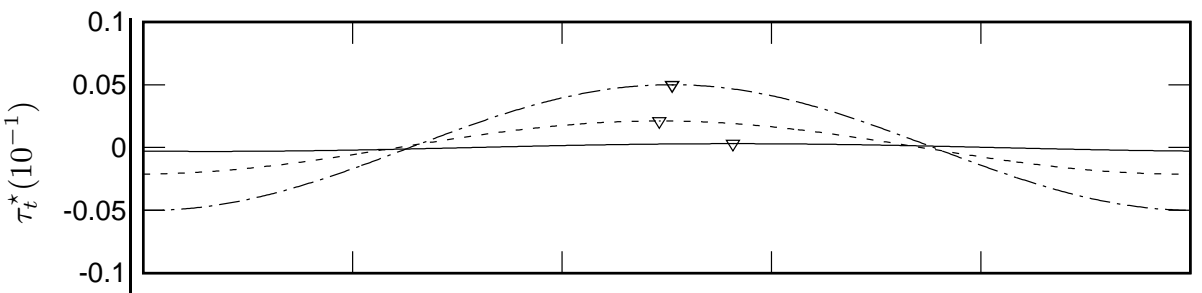

(c)

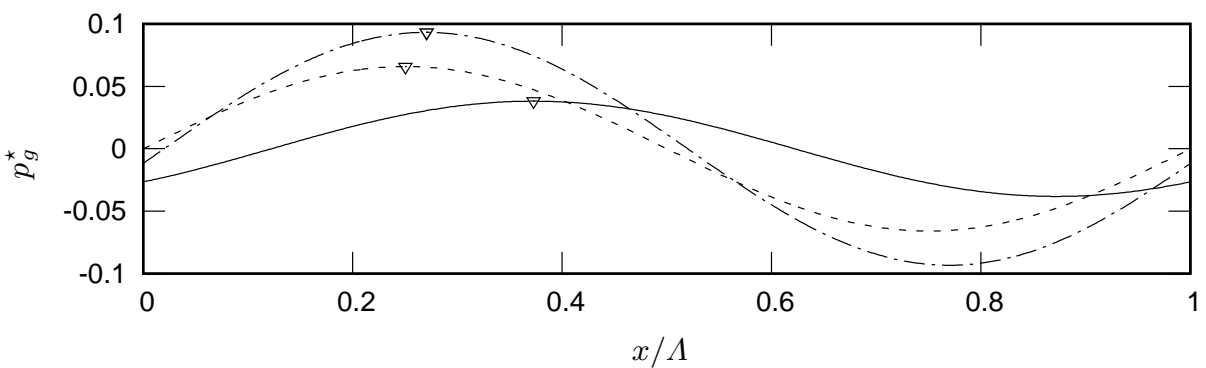

Figure 18. Water film at $\beta=3^{\circ}$ : linear perturbation of $(a)$ film thickness $(5.1),(b)$ tangential stress (5.3) and $(c)$ interfacial gas pressure (5.4) associated with a particular eigenmode, i.e. $\alpha=0.0025$ (wavelength $\Lambda=2 \pi / \alpha$ ). Solid line: $\eta=12.4$ aerostatic $(M=1)$; dashed line: $\eta=3.6$ aerostatic $(M=1)$; dash-dotted line: $\eta=3.6$ counter-current gas $(M=50)$. Triangles in panels $(b)$ and $(c)$ mark the location of the maximum. Pressure perturbations are scaled with different coefficients to facilitate the representation: 60 for $\eta=12.4 ; 600$ for $\eta=3.6$ aerostatic; 1500 for $\eta=3.6$ with $M=50$.

We quantify this by considering the linear perturbation amplitude of the inertialess flow rate (5.5) and by evaluating the contributions due to gravity $\hat{\mathrm{q}}^{g}$ (actually, this term also subsumes the effect of pressure) and shear stress $\hat{q}^{\tau}$ :

$$
\hat{\mathrm{q}}=\underbrace{-K_{l} h_{0}^{2} \hat{h}}_{\hat{\mathrm{q}}^{g}}+\underbrace{\frac{1}{2} h_{0}^{2} \hat{\tau}_{t}+h_{0} T_{t} \hat{h}}_{\hat{\mathrm{q}}^{\tau}} .
$$

Panels $(c)$ and $(d)$ of figure 19 show that the ratio $\left|\hat{\mathbf{q}}^{\tau} / \hat{\mathbf{q}}\right|$ increases with confinement and gas velocity, which is in accordance with the increase in stabilization observed in figures 11 and 14 .

The tangential shear stress also produces an indirect stabilizing effect. It is known that an adverse interfacial shear stress diminishes the celerity $c$ of surface waves traveling on falling films (Alekseenko et al. 2009; Samanta 2014). This can be explicitly shown in the limit of infinitely-long waves $(\alpha \rightarrow 0)$ :

$$
\left.c\right|_{\alpha \rightarrow 0}=-K_{l} h_{0}^{2}+T_{t} h_{0} .
$$

The wave celerity $c$ determines how fast the flow rate within the film would change in the inertialess limit as a wave passes by and reducing it weakens the inertial instability 
$(a)$

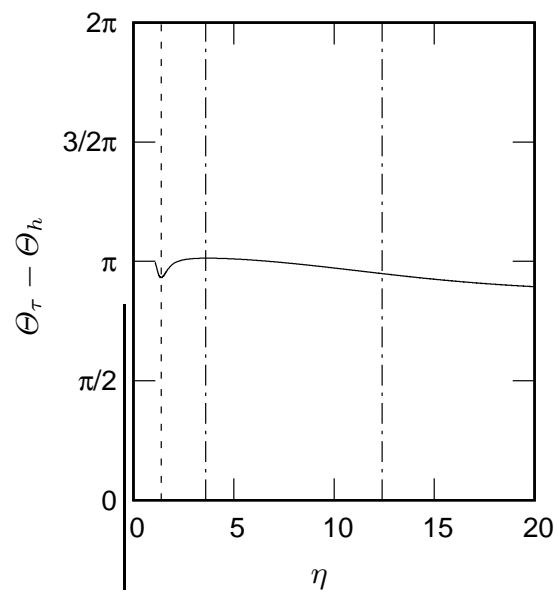

(c)

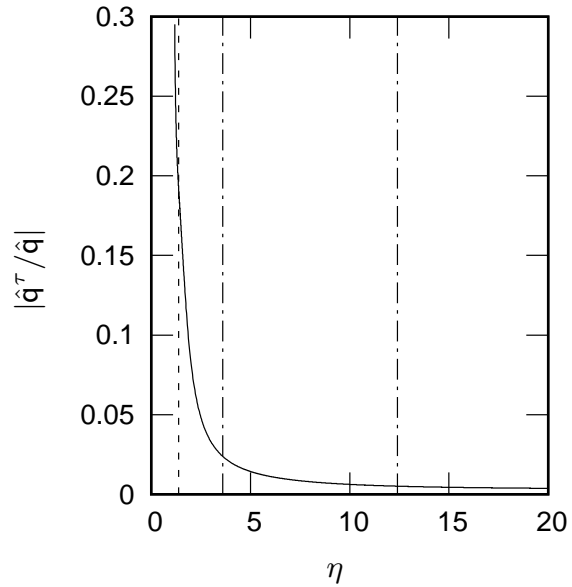

$(b)$

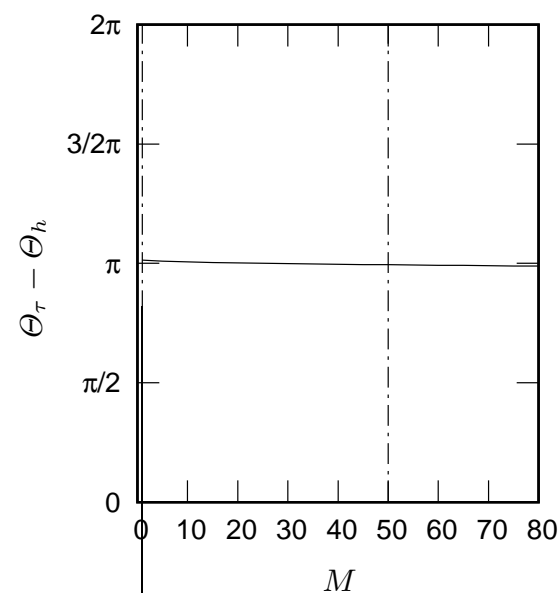

(d)

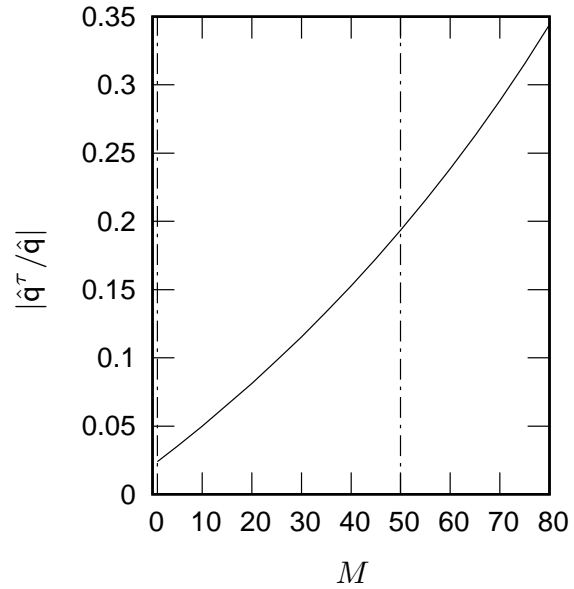

FiguRE 19. Water film at $\beta=3^{\circ}$ : phase lag $\Theta_{\tau}-\Theta_{h}$ of the perturbations of the film thickness and interfacial tangential shear stress. (a) Variation with $\eta$ with aerostatic gas $(M=1) ;(b)$ variation with $M$ at $\tilde{H}=1.87 \mathrm{~mm}(\eta=3.3-3.6)$. Contribution of the interfacial tangential shear stress to the inertialess flow rate (5.6): $(c)$ variation with $\eta$ under aerostatic gas $(M=1)$; $(d)$ variation with $M$ at $\tilde{H}=1.87 \mathrm{~mm}(\eta=3.3-3.6)$. The vertical dash-dotted lines mark the three cases represented in figure 18; the vertical dashed line in $(a)$ and $(c)$ marks $c_{r}=\left.U\right|_{h_{0}}$.

mechanism. In our stability results (figures $11 b$ and $14 b$ ), we have observed $c$ to diminish with increasing confinement and gas velocity, coinciding with an increased stabilization of the film.

\section{Conclusion}

Using linear stability calculations and experiments, we have revisited the stability of a falling liquid film flowing down an incline in interaction with a strongly-confined laminar gas flow and subject to the Kapitza instability. The gas is either subject to an aerostatic pressure gradient or flows counter-currently.

Tilley et al. (1994) suggested that confining the gas phase should stabilize the Kapitza instability, but concluded that this effect is small for water films in contact with air in the studied parameter range. For the same fluid combination, we have found a parameter range where the confinement-related stabilization is very strong, up to the point of 
suppressing the Kapitza instability altogether. We have shown that the stabilizing effect of confinement is stronger when the inclination is small $\left(\beta=1^{\circ}-8^{\circ}\right)$ and/or the Reynolds number is small, and we have determined the critical confinement necessary to fully stabilize the film as a function of these two parameters (figure 12). For example, a water film of $0.5 \mathrm{~mm}$ thickness flowing down a wall inclined at $3^{\circ}$ can be fully stabilized by placing a confining plate at a distance of $1 \mathrm{~mm}$ from the incline. Water films, which have a high Kapitza number, are found to feel earlier the confinement-induced stabilization than other working fluids with lower Kapitza number, such as aqueous solutions of glycerin or dymethylsulfoxide. The suppression of the Kapitza instability is observed both when the gas is subject to an aerostatic pressure gradient and when it flows counter-currently.

In the latter case, at the strong confinement levels we have focused on, the stabilization is monotonically intensified with increasing gas flow velocity and occurs over the entire range of unstable wavenumbers. This is different from observations by Alekseenko et al. (2009), Vellingiri et al. (2015), and Trifonov (2017), who found a non-monotonic effect of the gas velocity and an only partial destabilization of the wave spectrum for weaklyconfined turbulent gas flows. We have recovered these results also for the laminar gas flow conditions considered here, when reducing the confinement level. In this context, we have identified a confinement threshold above which the gas destabilizes the film at all wavenumbers (see figure 17).

We have identified the mechanism responsible for the instability suppression. As suggested by Tilley et al. (1994), viscous stresses in the gas play a decisive role. In particular, it is the tangential viscous stress exerted by the gas on the film surface that is decisive. The key of the stabilization mechanism is that there is a phase shift of almost exactly half a wavelength between the film thickness perturbation and the associated shear stress perturbation. Consequently, the adverse shear stress is greater at a wave hump than at a wave trough. This tends to homogenize the local-equilibrium flow rate within the liquid film, thus counter-acting the effect of gravity, which acts to force larger flow rates where the film is thick (wave hump) and smaller ones where the film is thin (wave trough). As a result, the inertia-related lag between the actual local flow rate and its equilibrium value is reduced and this weakens the inertia-driven mechanism of the Kapitza instability.

The stabilization mechanism increases whenever the contribution of the interfacial shear stress in the equilibrium flow rate becomes stronger with respect to that of gravity (and pressure). This is the case when the inclination is weak, the film is thin $\left(R e_{l}\right.$ is small) and the counter-current gas flow is strong. It is under those conditions that our stability calculations and experiments display the strongest stabilization.

We point out that confining the gas phase also contributes to the observed stabilization through an indirect effect, i.e. by reducing the wave celerity. Reducing the wave speed reduces inertia-induced flow rate imbalances within the liquid film, which again weakens the Kapitza instability.

\section{Acknowledgements}

This work was supported by the ANR wavyFILM project, grant ANR-15-CE06-001601 of the French Agence Nationale de la Recherche.

\section{Appendix A. Numerical validation of the stability code}

To validate our two-phase linear stability code numerically, we have compared its predictions to data from the literature for three examples of interfacial instability. 
(a)

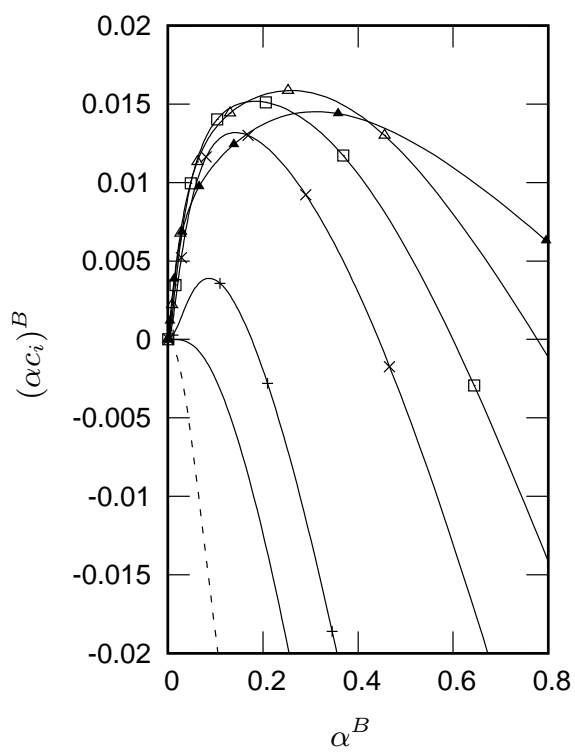

(b)

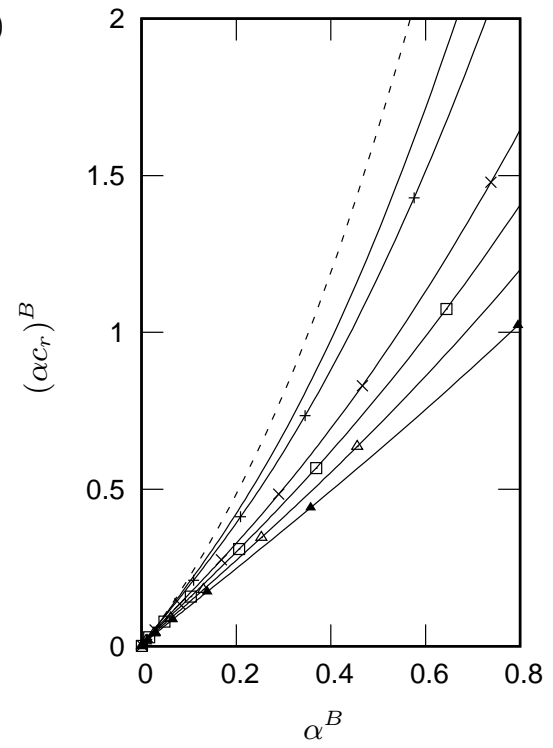

FiguRE 20. Comparison of (a) temporal growth rate and (b) angular wave frequency between our work and Brevdo et al. (1999) (figure 2 there). Parameter values: $\beta=4.6^{\circ}$, $\nu_{l}=5.02 \times 10^{-6} \mathrm{~m}^{2} \mathrm{~s}^{-1}, \gamma=69 \times 10^{-3} \mathrm{~N} \mathrm{~m}^{-1}, \rho_{l}=1130 \mathrm{~kg} \mathrm{~m} \mathrm{~m}^{-3}$, $K a=\gamma\left(\rho_{l} g^{1 / 3} \nu_{l}^{4 / 3}\right)^{-1}=331.85$. Values of the Reynolds number $R e^{B}=3 / 2 R e_{l}$ : 10 (dashed line), $R e_{c r}^{B}=5 / 4 \cot \beta$ (line), 20 (plus), 40 (cross), 60 (empty squares), 100 (empty triangles), 200 (filled triangles). Note that $\alpha^{B},\left(\alpha c_{r}\right)^{B}$ and $\left(\alpha c_{i}\right)^{B}$ are scaled as in Brevdo et al. (1999), i.e. using the Nusselt film thickness and the free-surface velocity as length and velocity scales.

Figure 20 compares our stability calculations with landmark calculations of Brevdo et al. (1999) for the case of an inclined liquid film falling in a passive atmosphere. We point out that the conditions studied by Brevdo et al. (1999) concern a liquid film flowing in a passive atmosphere $\left(\Pi_{\mu}=\Pi_{\rho}=0\right)$, whereas in the present work we have considered an active phase.

Figure 21 presents the neutral stability diagram for the long-wave Yih instability (horizontal pressure-driven two-layer flow with $\Pi_{\rho}=1$ ) as obtained by Yiantsios \& Higgins (1989), in comparison with our calculations (circles).

Finally, table 7 compares our stability data to those of Yiantsios \& Higgins (1988) and Tilley et al. (1994) for a pressure-driven two-layer flow through a horizontal channel, in the absence of gravity. In addition to the numerical punctual values of table 7 , we also present in figure 22 the real and imaginary part of the wave celerity as a function of the wavenumber for the case at $Q^{T}=1.6$ and $W e^{T}=0.2$ (forth and seventh row of table 7 ).

\section{Appendix B. Sensitivity of the comparison in figure 8 w.r.t. the inclination angle $\beta$}

To remedy the insufficient resolution of the inclinometer, the inclination angle $\beta$ of the experimental setup was obtained by fine-tuning (within the precision range $\pm 0.05^{\circ}$ of the inclinometer) the value of $\beta$ in the numerical calculation of the neutral stability curve for the unconfined case, until this matched with the corresponding experimental curve. Figure 8 shows the best match of these curves for one set of conditions, obtained for an inclination angle of $\beta=1.69^{\circ}$. 


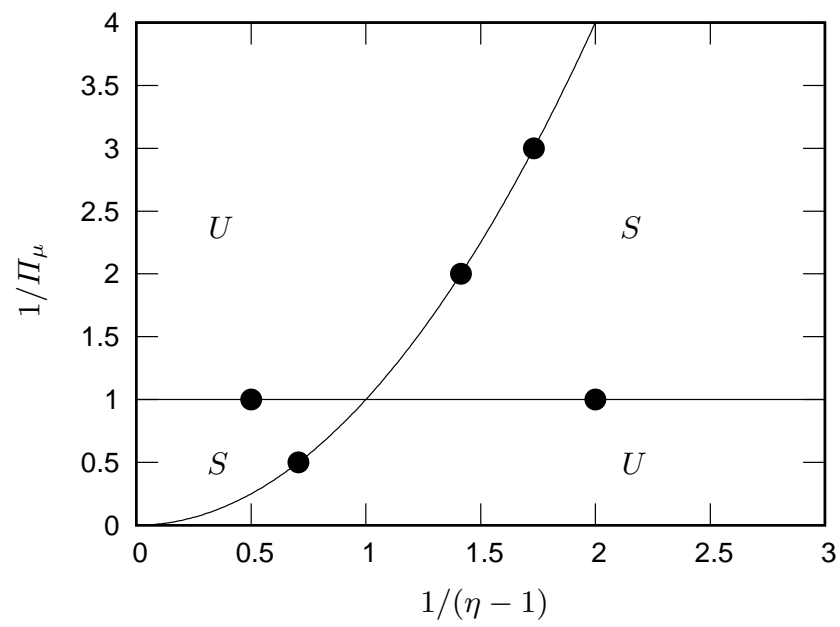

Figure 21. Neutral stability diagram for a horizontal pressure-driven two-layer flow with $\Pi_{\rho}=1$ : comparison of our numerical results (circles) with figure $2 \mathrm{~b}$ of Yiantsios \& Higgins (1989) (lines). Stable regions are denoted by $S$, unstable regions by $U$.

$\begin{array}{lllcccc}\alpha^{T} & Q^{T} & W e^{T} & \left(c_{r}, c_{i}\right)^{T}(\mathrm{YH}) & \left(c_{r}, c_{i}\right)^{T}(\mathrm{TDB}) & \left(c_{r}, c_{i}\right) H \mathcal{L}^{-1} \text { (this work) } \\ 20 & 0.32 & 0.016 & (0.399984,-0.0066148) & (0.399986,-0.0066151) & (0.399984,-0.0066151) \\ 20 & 0.32 & 0.008 & (0.399992,-0.0032796) & (0.399992,-0.0032796) & (0.399991,-0.0032798) \\ 20 & 1.6 & 0.4 & (1.99814,-0.166982) & (1.99815,-0.166982) & (1.99814,-0.166982) \\ 20 & 1.6 & 0.2 & (1.99912,-0.082368) & (1.99912,-0.082369) & (1.99912,-0.082369) \\ 40 & 0.32 & 0.008 & (0.3999976,-0.003325) & (0.3999977,-0.003325) & (0.3999974,-0.003325) \\ 40 & 0.32 & 0.004 & (0.3999988,-0.001658) & (0.3999989,-0.001658) & (0.3999985,-0.001658) \\ 40 & 1.6 & 0.2 & (1.99971,-0.083328) & (1.99971,-0.083329) & (1.99971,-0.083328) \\ 40 & 1.6 & 0.1 & (1.999858,-0.04150) & (1.999859,-0.04150) & (1.999857,-0.04150)\end{array}$

TABLE 7 . Real and imaginary part of the wave celerity $c: \Pi_{\mu}=1 / 5, \Pi_{\rho}=1$ and $\eta=2$. Comparison between our work and results of Yiantsios \& Higgins (1988) (table I there) and Tilley et al. (1994) (table I there). Scales used in Tilley et al. (1994) are related to ours as: $\alpha^{T}=\alpha H \mathcal{L}^{-1},\left(c_{r}, c_{i}\right)^{T}=\left(c_{r}, c_{i}\right) H \mathcal{L}^{-1}, Q^{T}=R e_{l}+R e_{g}, W e^{T}=W e H \mathcal{L}^{-1}$.

To demonstrate the sensitivity of this comparison w.r.t. the inclination angle, figure 23 shows this comparison for two slightly different values of $\beta$, i.e. $\beta=1.68^{\circ}$ (panel $a$ ) and $\beta=1.70^{\circ}$ (panel $\left.b\right)$.

\section{Appendix C. Measured streamwise evolution of the wave-amplitude}

Figure 24 shows the measured streamwise evolution of the wave amplitude along the channel for the "unconfined" (figure 4b) and the confined (figure 4a) configurations at $R e_{l}=38.7$, for the same inlet forcing amplitude and frequency. The film is unstable in both cases. The decrease of the wave amplitude induced by strengthening the confinement (changing $\eta=22$ to $\eta=6.65$ ) is approximately $7 \%$.

\section{REFERENCES}

Alekseenko, S. V., Aktershev, S. P., Cherdantsev, A. V., Kharlamov, S. M. \& 
(a)

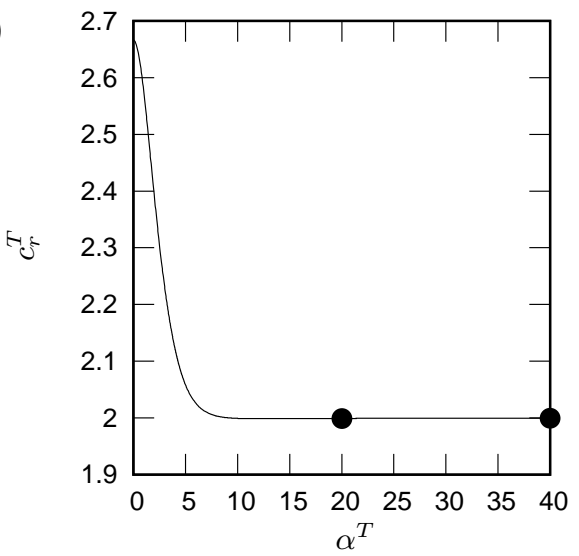

(b)

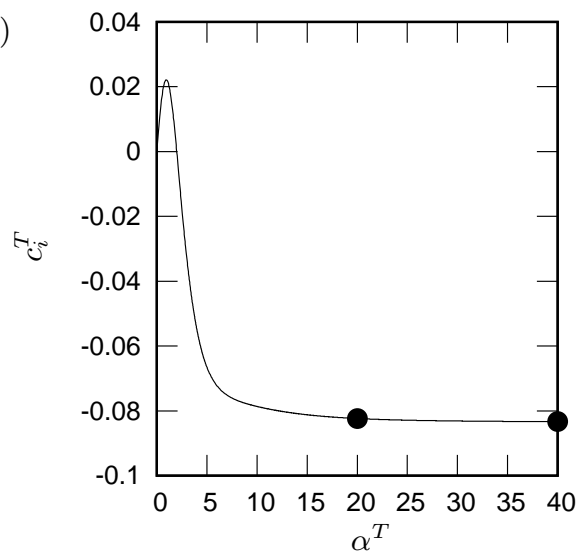

Figure 22. (a) Real and (b) imaginary wave celerity $c$ as a function of the wavenumber: $\Pi_{\mu}=1 / 5, \Pi_{\rho}=1, \eta=2, Q^{T}=1.6$ and $W e^{T}=0.2$ (same parameters as the forth and seventh row of table 7 , whose numerical values corresponding to our calculations are marked with filled circles). Scales used in Tilley et al. (1994) are related to ours as: $\alpha^{T}=\alpha H \mathcal{L}^{-1}$, $\left(c_{r}, c_{i}\right)^{T}=\left(c_{r}, c_{i}\right) H \mathcal{L}^{-1}, Q^{T}=R e_{l}+R e_{g}, W e^{T}=W e H \mathcal{L}^{-1}$.

(a)

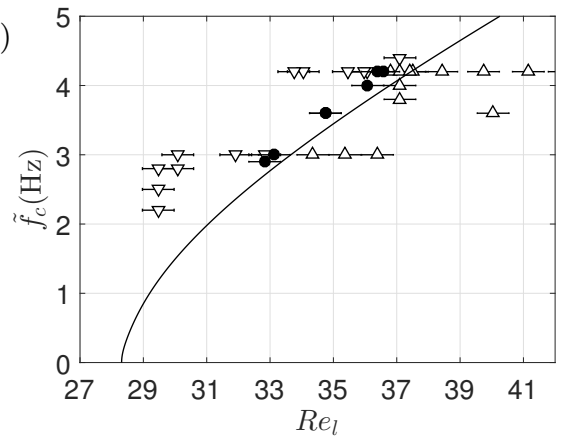

$(b)$

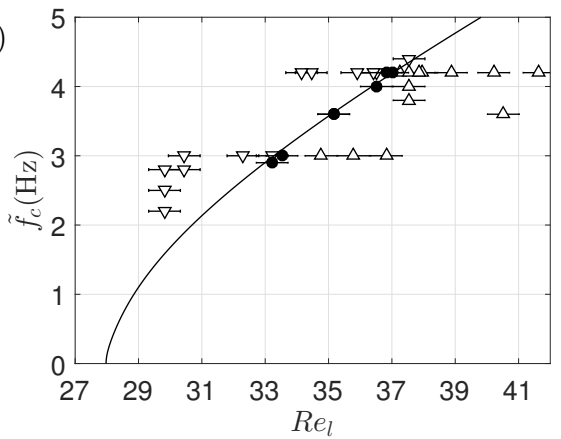

FIgURE 23. Sensitivity of the comparison in figure 8 w.r.t. the assumed inclination angle $\beta$ : (a) $\beta=1.68^{\circ} ;$ (b) $\beta=1.70^{\circ}$.

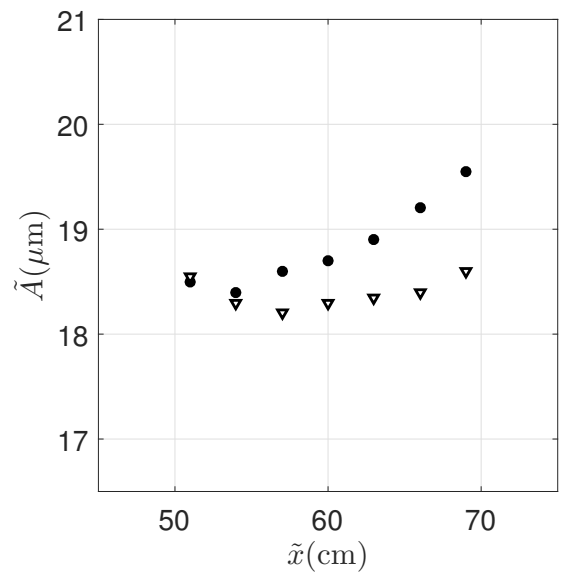

FiguRE 24. Experimental streamwise evolution of the wave amplitude for a water film at $R e_{l}=38.7$ and $\beta=1.69^{\circ}$ with aerostatic air. Open triangles: confined $(\eta=6.65)$; filled circles: "unconfined" $(\eta=22)$. Forcing frequency $\tilde{f}=3.6 \mathrm{~Hz}$. 
Markovich, D. M. 2009 Primary instabilities of liquid film flow sheared by turbulent gas stream. Int. J. Multiph. Flow 35, 617-627.

Alekseenko, S. V., Nakoryakov, V. Ye. \& Pokusaev, B. G. 1985 Wave formation on a vertical falling liquid film. AIChE J. 31 (9), 1446-1460.

Anshus, B. E. \& Goren, S. L. 1966 A method of getting approximate solutions to the orrsommerfeld equation for flow on a vertical wall. AIChE J. 12, 1004-1008.

Benjamin, T. B. 1957 Wave formation in laminar flow down an inclined plane. J. Fluid Mech. 2, 554-574.

Bergé, P., Pomeau, Y. \& Vidal, C. 1988 L'ordre dans le chaos, 5th edn. Hermann.

Boomkamp, P. A. M. \& Miesen, R. H. M. 1996 Classification of instabilities in parallel two-phase flow. Int. J. Multiph. Flow 22, 67-88.

Brevdo, L., Laure, P., Dias, F. \& Bridges, T. J. 1999 Linear pulse structure and signalling in a film flow on an inclined plane. J. Fluid Mech. 396, 37-71.

Cohen-Sabban, J., Gaillard-Groleas, J. \& Crepin, P.-J. 2001 Quasi-confocal extended field surface sensing. In Proceedings of SPIE (ed. A. Duparre \& B. Singh), Optical Metrology Roadmap for the Semiconductor, Optical, and Data Storage Industries II, vol. 4449, pp. $178-183$.

Dietze, G. F. 2016 On the Kapitza instability and the generation of capillary waves. J. Fluid Mech. 789, 368-401.

Dietze, G. F. \& Ruyer-Quil, C. 2013 Wavy liquid films in interaction with a confined laminar gas flow. J. Fluid Mech. 722, 348-393.

Doedel, E. J. 2008 AUTO-0\%p: continuation and bifurcation software for ordinary differential equations. Montreal Concordia University.

Floryan, J. M., Davis, S. H. \& Kelly, R. E. 1987 Instabilities of a liquid film flowing down a slightly inclined plane. The Physics of Fluids 30 (4), 983-989.

Gaster, M. 1962 A note on the relation between temporally increasing and spatially increasing disturbances in hydrodynamic stability. J. Fluid Mech. 14, 222-224.

Hinch, E. J. 1984 A note on the mechanism of the instability at the interface between two shearing fluids. J. Fluid Mech. 144, 463465.

Kalliadasis, S., Ruyer-Quil, C., Scheid, B. \& Velarde, M. G. 2012 Falling Liquid Films. Springer.

Kapitza, P. L. 1948 Wave flow of a thin viscous fluid layers. Zh. Eksp. Teor. Fiz. 18.

Kelly, R. E., Goussis, D. A., Lin, S. P. \& Hsu, F. K. 1989 The mechanism for surface wave instability in film flow down an inclined plane. Phys. Fluids A 1 (5), 819-826.

Kofman, N. 2014 Films liquides tombants avec ou sans contre-coulement de gaz : application au problme de l'engorgement dans les colonnes de distillation. PhD thesis, Université Pierre et Marie Curie.

Kofman, N., Mergui, S. \& Ruyer-Quil, C. 2017 Characteristics of solitary waves on a falling liquid film sheared by a turbulent counter-current gas flow. Int. J. Multiph. Flow 95, 2234.

Krantz, W. B. \& Goren, S. L. 1971 Stability of thin liquid films flowing down a plane. Ind. Eng. Chem. Fundamen. 10, 91-101.

Lavalle, G., Vila, J. P., Lucquiaud, M. \& Valluri, P. 2017 Ultraefficient reduced model for countercurrent two-layer flows. Phys. Rev. Fluids 2.

Liu, J., Paul, J. D. \& Gollub, J. P. 1993 Measurements of the primary instabilities of film flows. J. Fluid Mech. .

Ó NÁraigh, L., Spelt, P. D. M. \& Shaw, S. J. 2013 Absolute linear instability in laminar and turbulent gasliquid two-layer channel flow. J. Fluid Mech. 714, 58-94.

Pierson, F. W. \& Whitaker, S. 1977 Some theoretical and experimental observations of the wave structure of falling liquid films. Ind. Eng. Chem. Fundamen. 16, 401-408.

Ruyer-Quil, C. \& Manneville, P. 2000 Improved modeling of flows down inclined planes. The Eur. Phys. J. B 15, 357-369.

Samanta, A. 2014 Shear-imposed falling film. J. Fluid Mech. 753, 131-149.

Schmidt, P., Ó Náraigh, L., Lucquiaud, M. \& VAlluri, P. 2016 Linear and nonlinear instability in vertical counter-current laminar gas-liquid flows. Phys. Fluids 28. 
Smith, M. K. 1990 The mechanism for the long-wave instability in thin liquid films. J. Fluid Mech. 217, 469-485.

Tilley, B. S., Davis, S. H. \& Bankoff, S. G. 1994 Linear stability theory of two-layer fluid flow in an inclined channel. Phys. Fluids 6 (12), 3906-3922.

Trifonov, Y. Y. 2010 Counter-current gas-liquid wavy film flow between the vertical plates analyzed using the Navier-Stokes equations. AIChE J. 56 (8), 1975-1987.

Trifonov, Y. Y. 2017 Instabilities of a gas-liquid flow between two inclined plates analyzed using the NavierStokes equations. Int. J. Multiph. Flow 95, 144-154.

Valluri, P., Ó NÁraigh, L., Ding, H. \& Spelt, P. D. M. 2010 Linear and nonlinear spatiotemporal instability in laminar two-layer flows. J. Fluid Mech. 656, 458-480.

Vellingiri, R., Tseluiko, D. \& Kalliadasis, S. 2015 Absolute and convective instabilities in counter-current gasliquid film flows. J. Fluid Mech. 763, 166-201.

Whitaker, S. 1964 Effect of surface active agents on the stability of falling liquid films. Ind. Eng. Chem. Fundamen. 3, 132-142.

Yiantsios, S. G. \& Higgins, B. G. 1988 Linear stability of plane Poiseuille flow of two superposed fluids. Phys. Fluids 31 (11), 3225-3238.

Yiantsios, S. G. \& Higgins, B. G. 1989 Erratum: "Linear stability of plane Poiseuille flow of two superposed fluids" [Phis. Fluids 31, 3225 (1998)]. Phys. Fluids A 1 (5), 3225-3238.

Yıн, C. S. 1963 Stability of Liquid Flow down an Inclined Plane. Phys. Fluids 6 (3), 321-334.

Yıн, C. S. 1967 Instability due to viscosity stratification. J. Fluid Mech. 27, 337-352. 